\title{
TRAZAS FÓSILES DE TRILOBITES DE LA FORMACIÓN MOJOTORO (ORDOVÍCICO INFERIOR-MEDIO DE SALTA, ARGENTINA): IMPLICANCIAS PALEOECOLÓGICAS, PALEOBIOLÓGICAS Y BIOESTRATIGRÁFICAS
}

\author{
M. Gabriela MÁNGANO', Luis A. BUATOIS ${ }^{1} y$ \\ M. Cristina MOYA ${ }^{2}$ \\ Instituto Superior de Correlación Geológica. Casilla de Correo I (CC). 4000 \\ San Miguel de Tucumán. Argentina. ichnolog@infovia.com.ar \\ 2 Escuela de Geología. Universidad Nacional de Salta. Buenos Aires 177. 4400 \\ Salta. Argentina. crismoya@ciunsa.edu.ar
}

Mángano, M.G., Buatois, L.A. y Moya, M.C. 2001. Trazas fósiles de trilobites de la Formación Mojotoro (Ordovícico Inferior-Medio de Salta, Argentina): implicancias paleoecológicas, paleobiológicas y bioestratigráficas. [Trilobite trace fossils from the Mojotoro Formation (Lower to Middle Ordovician of Salta, Argentina): paleoecologic, paleobiologic and biostratigraphic implications.] Revista Española de Paleontología, 16(1), 9-28. ISSN ()213-6937.

\begin{abstract}
The Mojotoro Formation (Lower to Middle Ordovician, Salta, northwest Argentina) comprises thick packages of quartzites interbedded with fine-grained heterolithic facies, recording deposition in a tide-dominated shallowmarine setting. Depositional environments include subtidal sandwave complexes, interbars, bar margins, and tidal flats. Heterolithic facies hosts an abundant trilobite ichnofauna. The ichnogenus Cruzicina, represented by ichnospecies of the rugosa group (C. rugosa, C. furcifera, and C. goldfussi), is the dominant form, though Dimorphichnus has been recorded also. Trilobite traces of the Cruziana ichnofacies are cross-cut by vertical elements of the Skolithos ichnofacies representing palimpsest bedding surfaces. Thick packages of quartzites typically lack bioturbation due to strong tidal currents and highly mobile bedforms in the most active zones of subtidal sandwave complexes. Thinner beds representing deposition in smaller sandwaves and bar margins are dominated by Skolithos linearis, recording the activity of infaunal suspension feeders. Trilobite traces are abundant at the base of sandstone interbedded with mudstone layers or partings, recording outer zones of sandwave complexes, interbars, and intertidal flats. The high density of trilobite traces in heterolithic facies with sedimentary structures indicative of periodic emersion (e.g. desiccation cracks) is thought to record the feeding activities of trilobites in tidal flats. These periodic landward incursions were probably controlled by tidal cycles. The presence of the rugosa group and the absence of $C$. semiplicata indicate an Arenig-Llandeil age for the Mojotoro Formation, which is consistent with the age suggested by the faunal content of the underlying and overlying units.
\end{abstract}

Keywords: Trilobite trace fossils, trilobite paleoecology, Cruziana stratigraphy, Ordovician, tidal, shallowmarine, Gondwana, Argentina.

\section{RESUMEN}

La Formación Mojotoro (Ordovícico Inferior a Medio, Salta, noroeste de Argentina) comprende potentes paquetes de cuarcitas intercaladas con facies heterolíticas, registrando sedimentación en un ambiente litoral a marino somero dominado por mareas. Los ambientes sedimentarios incluyen complejos de ondas de arena submareales, zonas de interbarra, márgenes de barras y planicies de mareas. Las facies heterolíticas albergan una abundante icnofauna de trilobites. El icnogénero Crusiana, representado por las icnoespecies del grupo rugosa (C. rugosa, C. furcifiera y $C$. goldfisssi), es la forma dominante si bien se ha registrado también la presencia de Dimorphichnus. Las trazas de trilobites de la icnofacies de Cruziana están cortadas por elementos verticales de la icnofacies de Skolithos, representando superficies palimpsésticas. Los paquetes potentes de cuarcitas típicamente carecen de bioturbación muy posiblemente debido a las intensas corrientes mareales y a la alta movilidad de las 
formas de lecho en las zonas más activas de los complejos de ondas de arena. Las capas más delgadas, que representan sedimentación en ondas de arena más pequeñas y márgenes de barras, están dominadas por Skolithos linearis, registrando la actividad de suspensívoros infaunales. Las trazas de trilobites son abundantes en la base de areniscas intercaladas con capas o láminas pelíticas, registrando las zonas externas de los complejos de ondas de arena, interbarras y planicies intermareales. La alta densidad de trazas de trilobites en facies heterolíticas con estructuras indicativas de emersión (e.g. grietas de desecación) periódica registrarían las actividades de alimentación de trilobites en planicies de mareas. Estas incursiones periódicas hacia la costa fueron probablemente controladas por los ciclos mareales. La presencia del grupo rugosa y la ausencia de $C$. semiplicata indican una edad arenig-llandeilo para la Formación Mojotoro, lo cual es consistente con la edad sugerida por el contenido faunístico de las unidades infra y suprayacentes.

Palabras clave: Icnofósiles de trilobites, paleoecología de trilobites, estratigrafía de Cruziana, Ordovícico, mareal, marino somero, Gondwana, Argentina.

\section{INTRODUCCIÓN}

Las distintas icnoespecies del "grupo rugosa" (Seilacher, 1970), Cruziana rugosa, C. furcifera y $C$. goldfussi, presentan una amplia distribución perigondwánica. Este fue el primer grupo de Cruziana en ser descripto y es posiblemente el mejor caracterizado (Seilacher, 1992). Numerosos trabajos han registrado la presencia de integrantes de este grupo y discutido sus implicancias bioestratigráficas (e.g. Crimes y Marcos, 1976; Bergström, 1976; Pickerill et al. 1984; El-Khayal y Romano, 1988). Se han establecido zonaciones sobre la base de la icnoestratigrafía de Cruzicana en sucesiones paleozoicas de Gales, España, Polonia, norte de Africa, Sinaí, Jordania, Chad, Australia y Canadá, entre otras regiones. Sin embargo, muy pocos trabajos han intentado explorar las implicancias paleoecológicas de la presencia del grupo rugosa en facies cuarcíticas del Paleozoico Inferior.

Las trazas fósiles, en particular las estructuras biogénicas asignables a actividad de trilobites bentónicos, son elementos que han sido reiteradamente citados en sedimentitas del Paleozoico Inferior del noroeste argentino y Sierras Septentrionales de la provincia de Buenos Aires. Cabe señalar los pioneros estudios de Borrello (1966a,b), y posteriormente las contribuciones de Ramos (1973), Aceñolaza (1978), Aceñolaza y Durand (1978) y Aceñolaza y Fernández (1978), estas últimas mayormente centradas en el Ordovícico Inferior del noroeste argentino. A excepción de Aceñolaza (1978), quien sintetiza el conocimiento icnológico previo para el Paleozoico Inferior argentino desde una perspectiva estratigráfica e icnofacial, estos trabajos se centraron en la documentación de diversas icnoespecies de Cruzicana, Rusophycuss y otros icnogéneros en distintas unidades litoestratigráfícas del noroeste argentino.

En particular, Borrello (1966a) mencionó e ilustró la presencia de Cruziana furcifera $(C$. rugosa en este trabajo) en estratos ordovícicos de la quebrada de Gallinato, hoy asignables a la Formación Mojotoro (Moya, 1998). Volkheimer et al. (1980) reiteraron la presencia de Cruziana furcifera junto a Skolithos isp. y Palcieophycus isp. en estratos de la Formación Mojotoro, asignando esta unidad al intervalo ordovícico inferiorllanvirn basándose en una asociación microflorística. En el presente trabajo, se realiza una aproximación distinta al estudio de las trazas fósiles de trilobites, buscando analizar su vinculación con distintivas facies sedimentarias y ambientes de sedimentación de la Formación Mojotoro, de forma tal que se pueda extraer información sobre el modo de vida de los trilobites productores (cf. Mángano et cıl., 1999). En tal sentido, los objetivos de este trabajo son: (1) documentar las trazas fósiles asignables a la actividad de trilobites presentes en la Formación Mojotoro, (2) analizar la estrecha vinculación de las estructuras del grupo rugosc con facies costeras de planicie mareal y ambientes submareales adyacentes, de modo tal que permita explorar sus implicancias paleoecológicas, (3) evaluar la edad de la Formación Mojotoro basándose en la icnoestratigrafía de Cruziana y (4) discutir las posibles afinidades paleobiológicas del grupo rugosa.

\section{MARCO ESTRATIGRÁFICO Y SEDIMENTOLÓGICO}

La Formación Mojotoro, o Arenisca 4 de Moya (1998), aflora en la Sierra de Mojotoro (extremo sudoriental de la Cordillera Oriental), que se extiende al este de la ciudad de Salta (Fig. 1). Esta unidad se incluye en el Grupo Santa Victoria (Cámbrico Superior-Ordovícico Superior) (Fig. 2). La Formación Mojotoro se asigna al lapso arenig medio11 anvirn tardío, principalmente sobre la base de sus relaciones estratigráficas (Moya, 1998) y de su asociación de acritarcos (Volkheimer et cl., 1980). Hasta el presente no se ha hallado macrofauna diagnóstica en la Formación Mojotoro. La subyacente Formación San Bernardo contiene una variada fauna de graptolites, trilobites, braquiópodos articulados e inarticulados, bivalvos, cefalópodos, gasterópodos, crinoideos y ostrácodos, que sugieren una edad tremadoc tardía-arenig media (Harrington, 1938, 1957; Aceñolaza, 1973; Moya el (al., 1994; Moya, 1998). La suprayacente Formación Santa Gertrudis contiene una fauna diversa de conodontes, bivalvos, trilobites y braquiópodos articulados, que sugieren una edad llandeilo-caradoc temprana (Harrington, 1957: Monaldi y Monaldi, 1978; Monaldi, 1982: Sánchez, 1986; Albanesi y Rao, 1996; Waisfeld, 1996; Moya, 1998; Benedetto, 1999). Astini (1994) incluyó la Formación Mojotoro dentro de la 
Aloformación Sepulturas, criterio no adoptado por Moya (1998) en su revisión estratigráfica. Moya (1998) estimó en alrededor de $570 \mathrm{~m}$ el espesor total de la formación. Para el presente estudio se midieron secciones estratigráficas y se recolectó material icnofosilífero en las dos áreas en las que la Formación Mojotoro presenta mejores afloramientos, la quebrada de Gallinato y el río Mojotoro. En ambas localidades, la sucesión no es continua ya que se encuentra afectada por intenso fallamiento. La base de la Formación Mojotoro se encuentra cubierta en la quebrada de Gallinato y presenta un contacto por falla con la Formación San Bernardo en el río Mojotoro. El techo de la Formación Mojotoro es claramente observable en la quebrada de Gallinato, donde una superficie nítida la separa de la Formación Santa Gertrudis. En ambos afloramientos, la Formación Mojotoro está dominada por potentes paquetes de cuarcitas que alternan con intervalos heterolíticos de areniscas y pelitas. Si bien otros tipos de depósitos (facies pelíticas bioturbadas) se presentan asociados, restringiremos nuestra discusión a estas facies ya que son las más importantes volumétricamente y contienen las trazas fósiles analizadas en esta contribución.

Las cuarcitas son de grano fino, muy bien seleccionadas y se disponen en capas de 8 a $75 \mathrm{~cm}$ de espesor. Las capas de cuarcitas se apilan formando paquetes de hasta $40 \mathrm{~m}$ de potencia. La estructura primaria principal es la estratificación entrecruzada planar, tanto con capas frontales tangenciales como planares, si bien existen también capas con estratificación entrecruzada en artesa. El ángulo de inclinación de la estratificación entrecruzada planar varía de $15^{\circ}$ a $30^{\circ}$. Los paquetes de cuarcitas están limitados por superficies erosivas y planares de gran extensión y bajo ángulo. Internamente, se disponen superficies de orden menor, generalmente de mayor ángulo, tanto planares formando superficies de reactivación, como de geometría cóncava hacia arriba definiendo estratificación entrecruzada sigmoidal. Las pantallas de fango son escasas. Las capas frontales se encuentran inclinadas en una dirección predominante y sólo localmente se observan indicios de bidireccionalidad, representados por estratificación entrecruzada herringbone. Las trazas fósiles por lo general no son comunes en esta facies. Sin embargo, localmente pueden presentarse niveles con densas asociaciones de Skolithos linearis e incluso se ha detectado la presencia aislada de Cruziana en pequeñas artesas. Las trazas de trilobites son localmente abundantes en la base de algunas capas asociadas a láminas pelíticas. El dominio de estratificación entrecruzada planar indica la migración de formas de lecho de crestas rectas (bidimensionales). La presencia subordinada de estratificación entrecruzada en artesa sugiere la participación de formas de lecho de crestas sinuosas (tridimensionales). Los paquetes de cuarcitas se interpretan como acumulados en complejos de ondas de arena submareales localmente atravesados por canales. La estructura interna y la geometría de los cuerpos cuarcíticos son comparables con las descriptas para complejos de ondas de arena documentados en el registro estratigráfico (e.g. Bridges, 1982; Dalrymple, 1984; Stride, 1988; Santisteban y Taberner, 1988; Simpson y Eriksson, 1991; Bristow, 1995a, b).

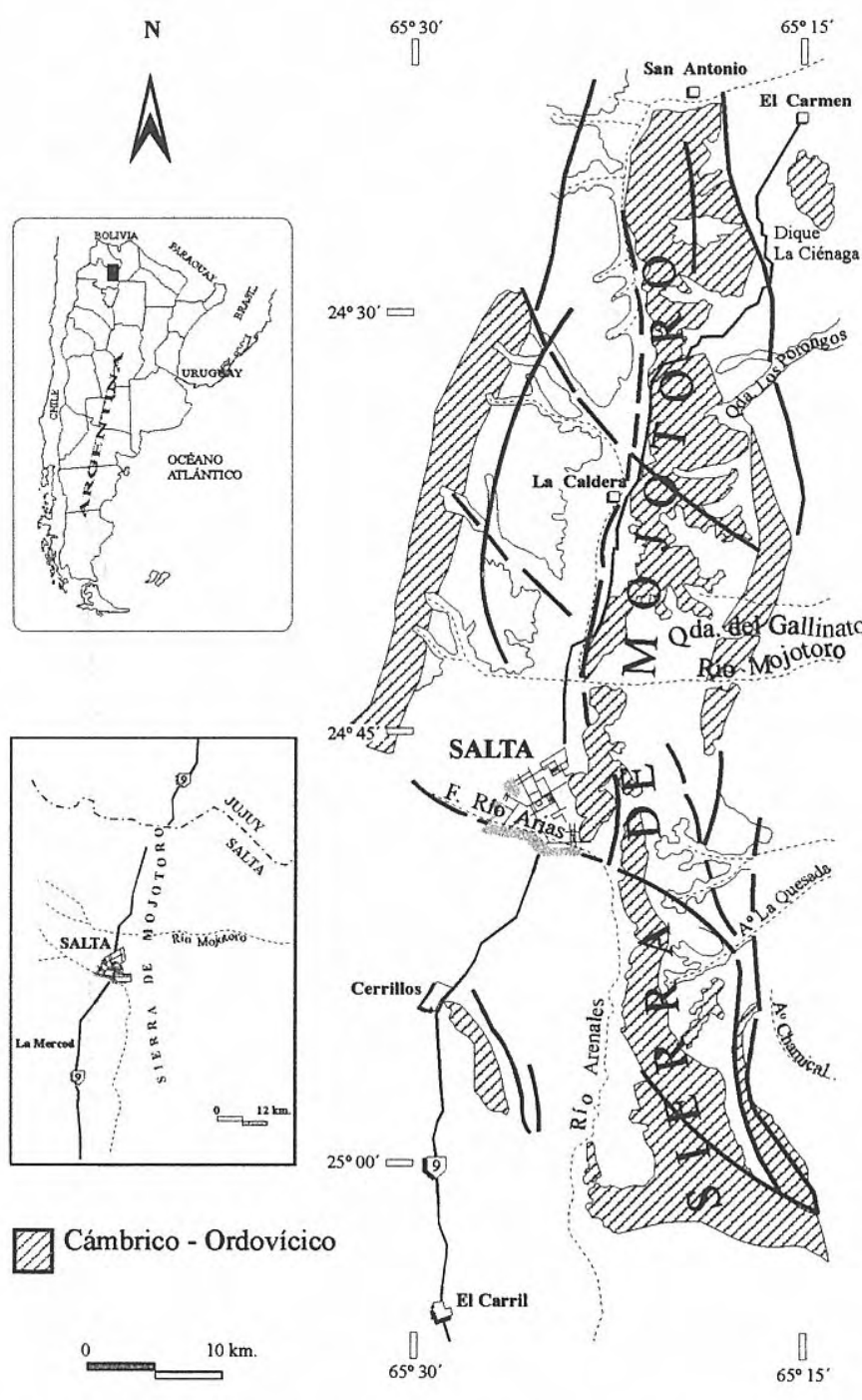

Figura 1. Mapa de la Sierra de Mojotoro mostrando la ubicación de los afloramientos del Paleozoico inferior. Modificado de Moya (1998).

Map of the Mojotoro Range showing the location of the Lower Paleozoic outcrops. Modified from Moya (1998).

Las facies heterolíticas comprenden delgadas intercalaciones de areniscas cuarzosas y wackes finas a muy finas, en capas de 1 a $17 \mathrm{~cm}$ de espesor, y limolitas en láminas de espesor milimétrico hasta capas de $17 \mathrm{~cm}$ de potencia. Estas intercalaciones forman intervalos de hasta 2,5 $\mathrm{m}$ de potencia. Pueden distinguirse dos tipos de facies heterolíticas. El tipo 1 se dispone en el tramo superior de sucesiones grano y estratodecrecientes representadas en su tramo inferior por las facies de cuarcitas previamente descriptas. A su vez, el intervalo de facies heterolíticas se presenta aquí en un arreglo granodecreciente, con dominio de capas arenosas y desarrollo de laminación ondulítica y estratificación flaser hacia la base y de estratificación ondulosa y lenticular hacia el tope. El techo de estas sucesiones es abrupto y está marcado por la base de un intervalo cuarcítico que marca el inicio de un nuevo ciclo. En este conjunto las areniscas presentan generalmente 


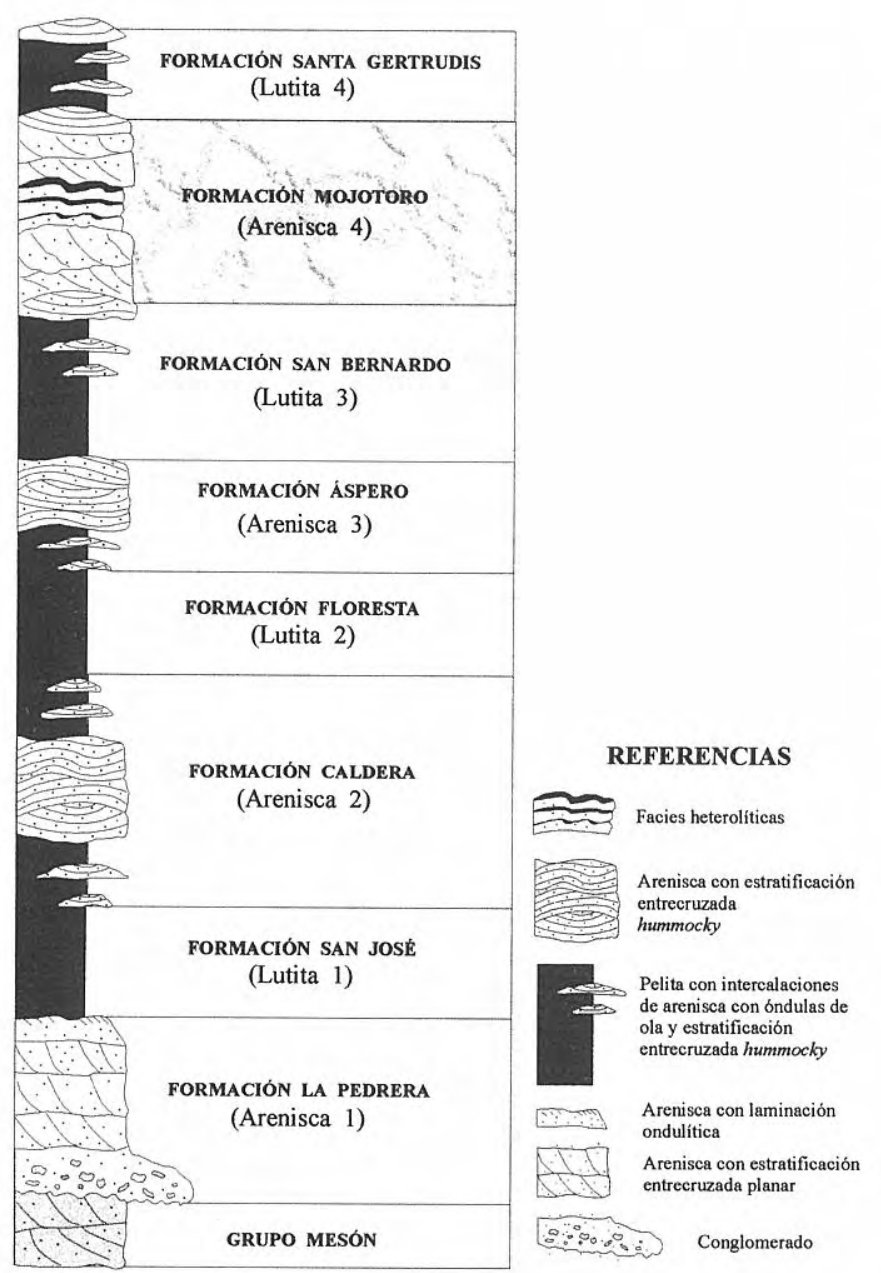

Figura 2. Estratigrafía del Grupo Santa Victoria (Cámbrico Superior-Ordovícico Superior) en la Sierra de Mojotoro. Unidades estratigráficas según la propuesta de Moya (1998).

Stratigraphy of the Santa Victoria Group (Upper Cambrian-Upper Ordovician) at the Mojotoro Range. Stratigraphic units after Moya (1998).

óndulas simétricas a cuasisimétricas y comúnmente desarrollan patrones de interferencia. Localmente se presentan asociadas grietas de desecación y de sinéresis. Las grietas de sinéresis presentan en planta típicas morfologías cuneiformes, desconectadas entre sí, mientras que las grietas de desecación se caracterizan por su geometría poligonal y mayor tamaño. Estos intervalos típicamente carecen de macrofauna asociada, con la excepción de restos de braquiópodos inarticulados que presentan una distribución localizada en distintos intervalos de la sucesión. Las trazas fósiles, por el contrario, suelen ser muy abundantes y mayormente se disponen en superficies densamente ocupadas por Cruziana, y ocasionalmente estructuras vermiformes tipo Palaeophycus, las cuales están cortadas por estructuras verticales profundas de Skolithos linearis y Arenicolites isp. En términos de estratigrafía secuencial, estas sucesiones representan típicas parasecuencias formadas por progradación de planicies mareales (Van Wagoner et al., 1990: fig. 3d). Parasecuencias de este tipo, mostrando el pasaje de ondas de arena submareales a planicies intermareales arenosas, mixtas y fangosas y pantanos supramareales, son analizadas en detalle por Klein (1971, 1977) y Dalrymple (1992). En el tipo 2, las facies heterolíticas se disponen o bien hacia la base de intervalos grano y estratocrecientes culminando en facies de cuarcitas o en ciclos simétricos donde se presentan entre intervalos cuarcíticos mediante relación gradacional. Las areniscas presentan óndulas simétricas y asimétricas y no se observan grietas de desecación asociadas. En estos intervalos se han hallado restos de braquiópodos inarticulados y fragmentos de trilobites. Estas facies heterolíticas contienen tanto trazas de trilobites como Skolithos linearis. Este segundo conjunto representaría los depósitos acumulados en zonas de menor energía dispuestas entre los cuerpos de ondas de arena. Sucesiones de este tipo son menos conocidas en el registro, probablemente porque los depósitos de interbarra tienden a ser erosionados por la migración de las ondas de arena. En ambientes actuales, mantos de arena y fango se disponen en las zonas laterales o terminales de los sectores con transporte neto de arena tanto en plataformas continentales como en los márgenes externos de estuarios (Bridges, 1982). Ejemplos de intercalaciones heterolíticas acumuladas en ambientes de este tipo han sido documentados en el registro estratigráfico por Anderton (1976). Sucesiones grano y estratocrecientes vinculadas al establecimiento de cuerpos arenosos submareales fueron descriptas en el Cretácico del oeste de Estados Unidos, pero asociadas al desarrollo de bancos de arena y no de complejos de ondas de arena (e.g. Berven, 1966). Existen, sin embargo, dudas sobre esta interpretación (cf. Bridges, 1982). Ciclos simétricos producidos por el establecimiento y posterior abandono de un complejo de ondas de arena fueron documentados por Hein (1987) en el Cámbrico de Canadá.

En síntesis, los depósitos de la Formación Mojotoro se interpretan como acumulados en un ambiente litoral y marino somero dominado por la acción de mareas. La magnitud de los depósitos de ondas de arena analizados sugiere un régimen de tipo macromareal. Complejos de ondas de arena de este tipo se desarrollan en ambientes de plataforma o en sectores restringidos donde se produce una amplificación del rango mareal, tales como estrechos (e.g. McCave, 1971) y estuarios (e.g. Dalrymple, 1984). La escasez de cuerpos fósiles en el intervalo analizado contrasta con la abundante macrofauna presente en las unidades infra y suprayacentes, lo cual sugiere condiciones ecológicas rigurosas (cf. Astini, 1994). Asimismo, la icnodiversidad de la Formación Mojotoro es relativamente baja, hecho que indicaría condiciones de estrés ambiental (cf. Mángano y Buatois, 1997). Este hecho sería compatible con un ambiente de valle estuarino como el sugerido por Astini (1994) para la Aloformación Sepulturas. La base de la Formación Mojotoro no es observable en las localidades analizadas por lo que no ha podido comprobarse la existencia de una superficie de incisión estuarina. En otras localidades, Moya (1998) mencionó un pasaje gradual entre las formaciones San Bernardo y Mojotoro. Por su parte, el contacto entre las formaciones Mojotoro y Santa Gertrudis es neto y, de adoptarse un modelo estuarino, se interpretaría como una superficie de ravinamiento de oleaje que separa facies de barra de boca de estuario de facies marinas abiertas 
portadoras de una fauna indicadora de salinidad normal. Las numerosas fallas que afectan a la sucesión y la cobertura vegetal hacen muy difícil estimar el espesor verdadero de la secuencia analizada.

\section{ICNOTAXONOMÍA}

\section{FILOSOFÍA ICNOTAXONÓMICA}

Las trazas fósiles constituyen la expresión sedimentaria de las funciones de comportamiento de antiguos organismos (Frey y Wheatcroft, 1989). Desde esta perspectiva, el fundamento último de la icnotaxonomía debe ser el comportamiento representado. En tal sentido, Cruziana, Rusophycus y Dimorphichnus, por citar algunos icnogéneros, constituyen icnotaxones de alto rango, basados en amplios grupos etológicos de fácil reconocimiento (i.e. categorías etológicas). El comportamiento se manifiesta en los rasgos morfológicos de las estructuras biogénicas que el icnólogo debe interpretar basándose en su conocimiento de patrones de comportamiento y morfología funcional del posible grupo de organismos en cuestión. En esta tarea de desciframiento deben filtrarse aquellos rasgos morfológicos impartidos por procesos tafonómicos, ajenos a la actividad del organismo. Existe una tendencia creciente en icnotaxonomía a considerar que el mero aspecto superficial de la traza, su morfología aparente, no constituye criterio suficiente para nombrar una estructura biogénica (Uchman, 1998; Rindsberg, 1999). El entendimiento del modo de producción y la función de una estructura permite evaluar qué caracteres morfológicos son significativos y cuáles triviales. Este discernimiento puede ser ciertamente una ardua tarea, pero las dificultades en la reconstrucción de la estructura en cuestión o en el entendimiento de cómo fue generada no deberían influenciar los principios básicos de la icnotaxonomía (Uchman, 1998).

Esta filosofía icnotaxonómica es aquí aplicada al estudio de las actividades de trilobites en los depósitos litorales y marinos someros de la Formación Mojotoro. Un gran número de icnogéneros han sido atribuidos a las distintas actividades desarrolladas por trilobites bentónicos, incluyendo Cruziana, Rusophycus, Diplichnites, Petalichnus, Asaphoidichnus, Dimorphichnus y Monomorphichnus, entre otros. Seguimos la propuesta de distintos autores de retener Rusophycus para estructuras bilobuladas, someras a relativamente profundas, que registran excavación localizada, más o menos en posición estática por parte de apéndices, pudiendo participar otros elementos morfoestructurales, tales como el céfalo, (e.g. Crimes, 1970a,b; Osgood, 1970; OrXowski et al., 1971; Bergström, 1973, 1976). Estas estructuras pueden cumplir distintos propósitos, tales como descanso temporario, cámaras de habitación, nidificación o predación (Fenton y Fenton, 1937; Seilacher, 1955; Osgood, 1970; Jensen, 1990). El icnogénero Cruziana corresponde a excavaciones acintadas, subsuperficiales a relativamente profundas, interpretadas comúnmente como estructuras de locomoción por acción sincronizada de múltiples apéndices o de locomoción combinada con alimentación (pascichnia) (Crimes, 1970b; Seilacher, 1955, 1970). Otros icnogéneros que comúnmente representan estilos de locomoción o alimentación, vinculados a las actividades bentónicas de trilobites, son Diplichnites, que registra locomoción a pasos (walking), Dimorphichnus, producido por el desplazamiento lateral del animal sobre el substrato, y Monomorphichnus, interpretado como una combinación de natación y pastoreo. Dimorphichnus ha sido interpretada como una estructura de pastoreo en la cual se utilizan asimétricamente los apéndices de ambos lados del cuerpo del organismo produciendo marcas de empuje (pushing marks) y de rastrillado (raking marks) (cf. Seilacher, 1955, 1990). El icnogénero Monomorphichnus fue creado por Crimes (1970b) para incluir marcas de rasguñaduras monomórficas que presentan repetición lateral. Estas estructuras fueron interpretadas por dicho autor como estructuras de pastoreo producidas por el rastrillaje de los apéndices de un sólo lado del cuerpo al tiempo que el organismo era desplazado por una corriente (cf. Crimes, 1970b: fig. 6). Trabajos posteriores han utilizado Monomorphichnus básicamente para describir sets repetidos de marcas de rasguñaduras que no muestran una disposición bilateral (e.g. Crimes et al., 1977; Crimes y Anderson, 1985; Fillion y Pickerill, 1990; Mángano et al., 1996b), basando las distintas icnoespecies principalmente en la morfología de las marcas de rasguñaduras o su patrón de ordenamiento (e.g. M. linearis, M. multilinearis, $M$. intersectus). Monomorphichnus es un icnogénero cuyo reconocimiento es problemático y su significado etológico cuestionable. Osgood $(1970,1975)$ notó que, si Dimorphichnus y Monomorphichnus son interpretadas como estrategias de alimentación, éstas serían sumamente ineficientes y no habrían sido utilizadas por trilobites después del Cámbrico. Alternativamente, estos icnogéneros registrarían el resultado del accionar del trilobites sobre el substrato al ser arrastrado por una corriente (Martinsson, 1965; Osgood, 1970). Por su parte, Seilacher (1985, 1990) sostuvo que Monomorphichnus corresponde a una forma preservacional de Dimorphichnus, explicando el primero como una subtraza (undertrace) que registra parcialmente la morfología completa de la estructura (cf. Goldring y Seilacher, 1971). El reconocimiento de Dimorphichnus presenta ciertas dificultades de índole tafonómica: (1) su frecuente preservación como crestas hipicnias formando densas superficies con alto grado de superposición y (2) la reconstrucción completa de la estructura suele requerir de amplias superficies. Estas dificultades deben ser subsanadas por medio de estudios detallados que tengan en cuenta la naturaleza de estas subtrazas y contando con una cantidad de material que pueda evaluarse como representativa. La distinción entre Dimorphichnus y Monomorphichnus, cuando se cuenta con material muy fragmentario, puede ser en la práctica imposible.

El material recolectado puede inscribirse casi en su totalidad en el icnogénero Cruziana y, en particular, en el 
denominado grupo rugosa. Estos "grupos" fueron originalmente establecidos basándose en semejanzas morfológicas, pero no deben entenderse como una categoría icnotaxonómica. El reconocimiento de distintas icnoespecies de Cruziana y Rusophycus se basa fundamentalmente en la morfología y agrupamiento de las marcas de rasguñaduras, teniendo también en cuenta la técnica de excavación y filtrando todo efecto tafonómico (cf. Seilacher, 1970; Bergström, 1973, 1976). En tal sentido, las tres icnoespecies reconocidas para el grupo rugosa registradas en la Formación Mojotoro, $C$. rugosa, $C$. furcifera y $C$. goldfussi, atestiguan tres técnicas de instrumentación de apéndices y, como tales, constituyen icnotaxones con reconocible significado etológico. La mayor parte de los estudios sobre estructuras cruzianiformes del grupo rugosa reconocen las tres icnoespecies antes mencionadas, destacando sus diferencias morfológicas y su significación estratigráfica. El rango estratigráfico de las tres icnoespecies del grupo rugosa no es exactamente coincidente (cf. Seilacher, 1970, 1992; Baldwin 1977a; Crimes 1970a; Pickerill et al., 1984; El-Khayal y Romano, 1988; Fillion y Pickerill, 1990). Por ejemplo, Cruziana furcifera parece presentar cierta superposición con el rango de $C$. semiplicata, en ausencia de Cruziana rugosa (Crimes, 1970a, 1975a, b; Baldwin, 1977a; Pickerill y Fillion, 1983; Crimes y Marcos, 1976). Sobre la base del estudio de 150 especímenes provenientes del Ordovícico Inferior del noreste de España, Kolb y Wolf (1979) sinonimizaron $C$. furcifera, C. goldfussi, C. breadstoni, C. tortworthi y $C$. barriosi con $C$. rugosa por regla de prioridad. Este estudio adolece de serios problemas metodológicos (cf. Pickerill et al., 1984: p. 255-256). A su vez, y más importante aún, los fundamentos sobre los cuales dichos autores edifican la sinonimia, transicionalidad y ausencia de valor estratigráfico, son incorrectos. El hecho de que estos icnotaxones puedan presentar relaciones intergradacionales entre sí no constituye un argumento válido para sinonimizarlos (cf. Bromley y Frey, 1974; Pickerill y Narbonne, 1995). De igual modo, el potencial bioestratigráfico, si bien deseable, no constituye en sí mismo una sólida base para fundar una icnotaxonomía. Una situación inversa ocurre con Cruziana semiplicata. Esta icnoespecie engloba un amplio rango de morfotipos que evidencian distintas técnicas de excavación en posición prosoclina, isoclina y opistoclina (cf. Seilacher, 1970: lám. 1, a-g). Llamativamente, Cruziana semiplicata también parece mostrar ciertas variaciones en su morfología a lo largo de su rango estratigráfico, cámbrico superior-tremadoc, pero no ha sido subdividida en distintas icnoespecies.

Otro punto interesante de notar es que las diferencias morfoetológicas entre dos icnoespecies de Cruziana correspondientes a distintos grupos (e.g. C. rugosa contra C. semiplicata) son comúnmente de mayor envergadura que aquellas que diferencian icnoespecies del mismo grupo (e.g. $C$. rugosa contra $C$. furcifera). Una de las posibles propuestas para mantener la consistencia interna del sistema icnotaxonómico establecido, sin perder información etológica, sería la adopción de icnosubespecies. De este modo, las icnoespecies del grupo rugosa pasarían al rango de icnosubespecies: Cruziana rugosa rugosa, Cruziana rugosa furcifera y Cruziana rugosa goldfussi (cf. Seilacher, 1996: p. 529). Como contraparte, sería necesario establecer formalmente icnosubespecies de C. semiplicata. Del mismo modo, Seilacher (1996) reconoció cuatro icnosubespecies de Cruziana acacensis: Cruziana acacensis plana, Cruziana acacensis retroversa, Cruziana acacensis sandaliana y Cruziana acacensis laevigata basándose en modificaciones de la técnica de excavación. Dado que las icnosubespecies no han sido formalmente incluidas en la última edición del ICZN (1999; versión en español, 2000), preferimos tratar a las formas del grupo rugosa como icnoespecies, una vez hecha la acotación precedente.

\section{DESCRIPCIONES SISTEMÁTICAS}

El material se encuentra despositado en las colecciones de paleontología de invertebrados de la Facultad de Ciencias Naturales e Instituto Miguel Lillo de la Universidad Nacional de Tucumán.

\section{Icnogénero Cruziana d’Orbigny, 1842}

\section{Cruziana furcifera d’Orbigny, 1842 Fig. 3}

Material: once especímenes en las muestras número PIL 12817, PIL 12818, PIL 12819, PIL 11789, PIL 14615, PIL 14616, PIL 14626, PIL 14628, PIL 14629, PIL 14635, y aproximadamente quince ejemplares observados en el campo.

Figura 3. Cruziana furcifera. a. Superposición de numerosos especímenes acintados, de escasa profundidad, asignables a las icnoespecies C. furcifera y C. goldfussi. Numerosos especímenes de Skolithos linearis cortando los especímenes de Cruziana, representando una fábrica palimpséstica. PIL 12258. b. Cruziana furcifera en transición con C. rugosa en el sector anterior. PIL 14628. c. Cruziana furcifera. Nótese típicos entrecruzamientos en ángulo agudo de sets de marcas de rasguñaduras característicos de esta icnoespecie (mejor desarrollados en el lóbulo derecho) y Skolithos linearis cortando Cruziana. PIL 14629. Barra $=1 \mathrm{~cm}$.

Cruziana furcifera. a. Superposition of several ribbon-like, shallow specimens of C. furcifera and C. goldfussi. Various specimens of Skolithos linearis are cross-cutting specimens of Cruziana, representing a palimpsest fabric. PIL 12258. b. Cruziana furcifera transitional with C. rugosa at the anterior part. PIL 14628. c. Cruziana furcifera. Note typical scratch marks criss-crossing at acute angle characteristic of this ichnospecies (best developed on the right lobe), and Skolithos linearis cross-cutting Cruziana. PIL 14629. Bar $=1 \mathrm{~cm}$. 

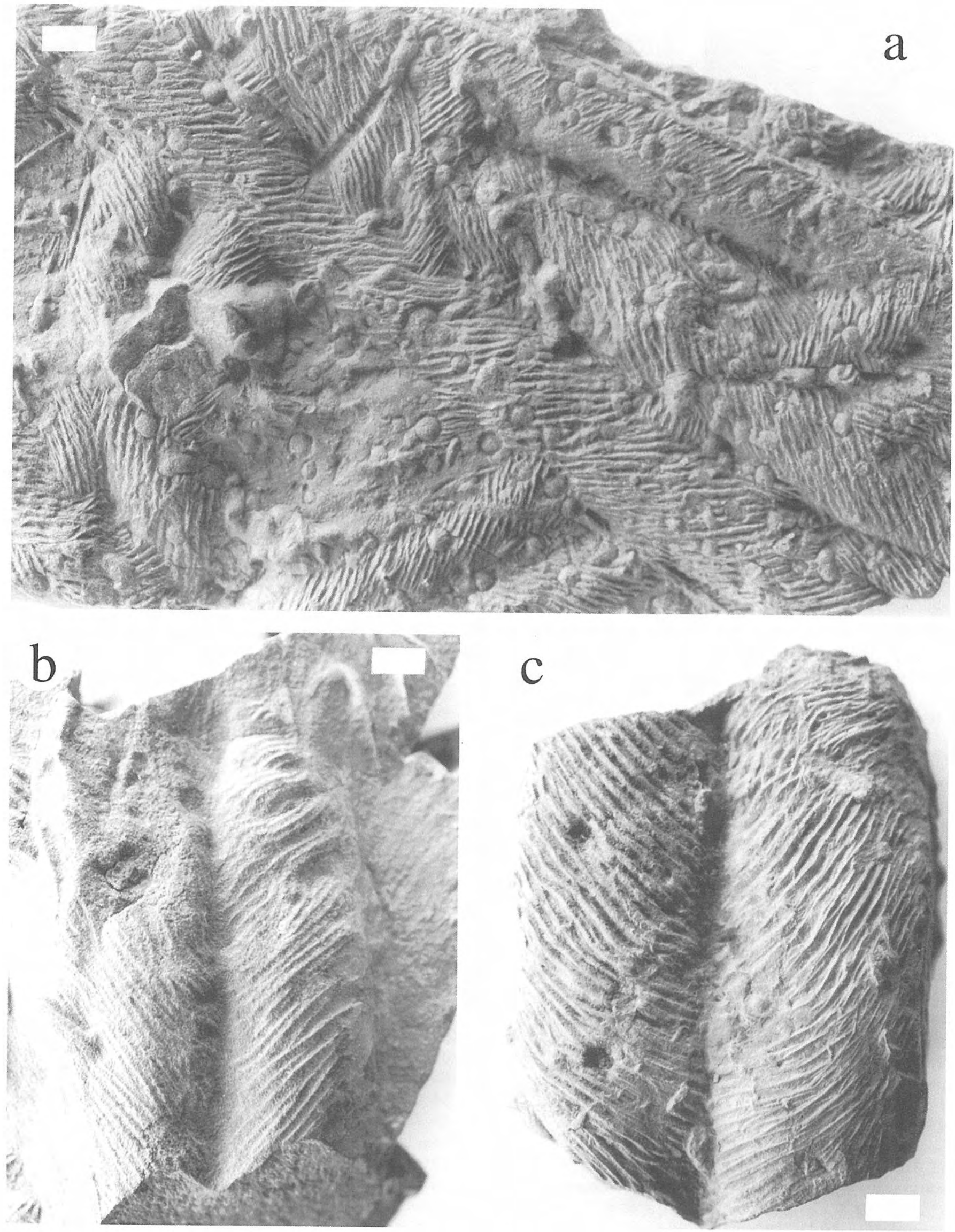

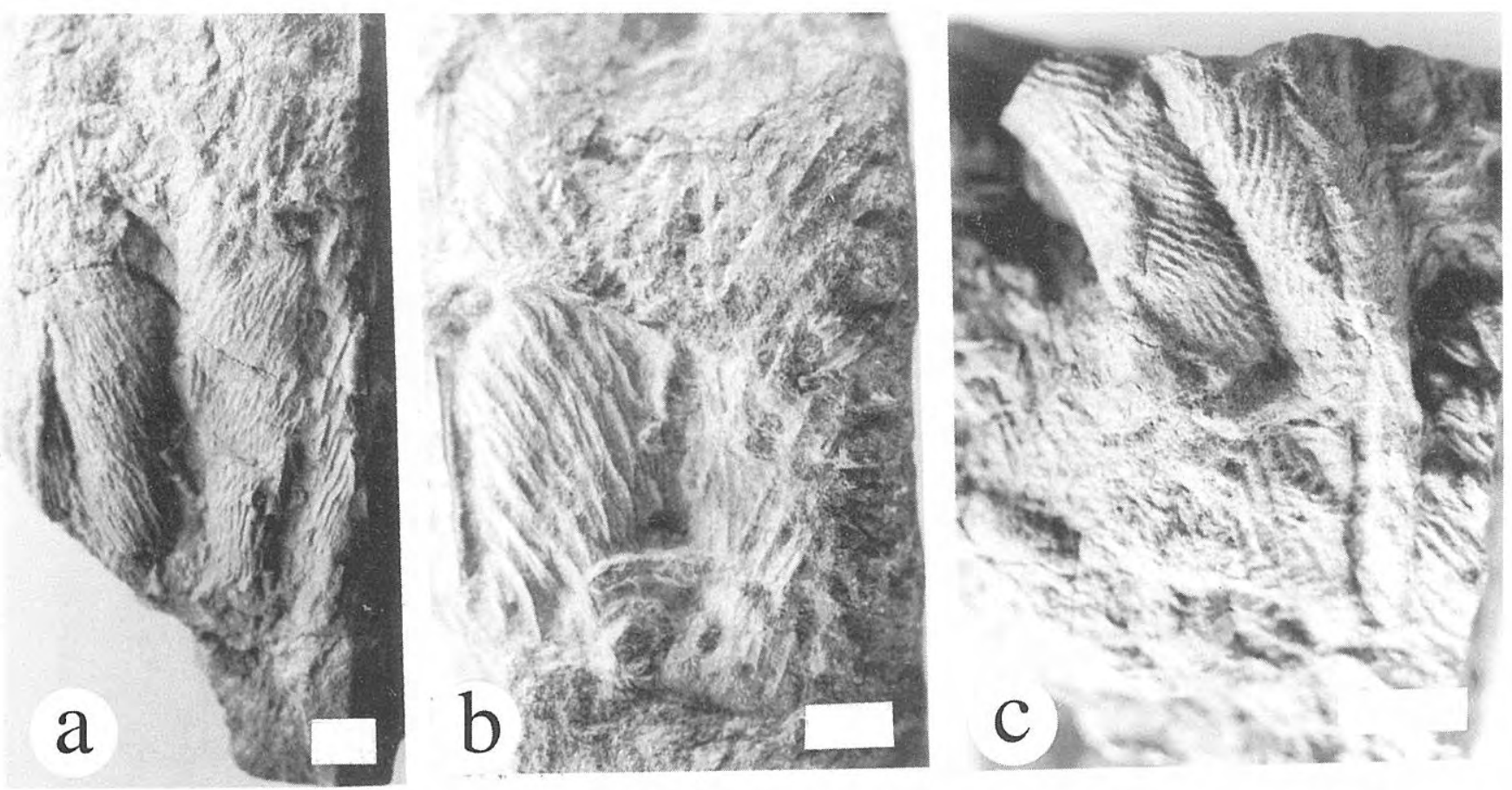

Figura 4. Cruziana goldfussi. a. Parte posterior de un ejemplar en forma de cubeta en "U". Nótese surco marginal discontinuo y finas marcas de rasguñaduras endopodales características de esta icnoespecie. PIL 14641. b. Espécimen mostrando tendencia de las marcas de rasguñaduras endopodales a orientarse paralelas al surco medio posteriormente. Surco marginal bifurcado claramente visible en el lóbulo izquierdo. PIL 12818. c. Ejemplar pequeño mostrando un surco marginal prominente (lóbulo derecho). Barra $=1 \mathrm{~cm}$.

Cruziana goldfussi. a. Posterior part of a specimen showing U-shaped bathtube morphology. Note discontinuous marginal furrow and fine endopodial scratch marks characteristic of this ichnospecies. PIL 14641. b. Specimen showing endopodial scratch marks that tend to display parallel orientation with respect to the median furrow posteriorily. Bifurcated marginal furrow clearly visible on the left lobe. PIL 12818. c. Small specimen showing a prominent marginal furrow (right lobe). PIL 14607. Bar $=1 \mathrm{~cm}$.

\section{Descripción}

Crestas hipicnias bilobuladas rectas o levemente curvadas, con marcas de rasguñaduras retroversas que cubren la totalidad de los lóbulos formando una "V". El ancho varía entre 43,2 y 58,3 $\mathrm{mm}$. La máxima longitud registrada en un ejemplar es de $167,5 \mathrm{~mm}$. La profundidad de la traza varía entre 19,2 y $31,8 \mathrm{~mm}$. Surco medio recto bien definido. Crestas marginales típicamente ausentes, si bien ocasionalmente pueden presentarse en especímenes transicionales con $C$. goldfussi (Fig. 3a). Marcas de rasgado típicamente angulosas, agrupadas en sets múltiples, poco definidos, de hasta 8 marcas. Los sets sucesivos se entrecruzan formando un ángulo agudo y definiendo localmente áreas romboidales (diamond-shaped areas). El ángulo de la "V" varía entre $60^{\circ}$ y $115^{\circ}$. El ángulo formado por las marcas de rasguñaduras puede variar a lo largo de una misma traza, tendiendo a disminuir hacia el sector posterior de la misma.

\section{Discusión}

Cruziana furcifera, C. rugosa y $C$. goldfussi integran el denominado "grupo rugosa" y representan variedades etológicas vinculadas a distintos modos de excavación (Seilacher y Crimes, 1969; Seilacher 1970, 1992; Bergström, 1973, 1976; Baldwin, 1977a). Cruziana furcifera registraría la acción retroversa de los endopoditos en posición opistoclina (Seilacher, 1970, 1992). En su estudio sobre la Serie de Los Cabos, en España, Baldwin (1977b) notó que Cruziana furcifera suele presentar márgenes de suaves pendientes e interpretó esta icnoespecie como una variedad menos profunda y de avance más veloz que la documentada por C. rugosa. En la Formación Mojotoro, C. furcifera, al igual que $C$. goldfussi, suele presentar lóbulos planos a levemente convexos y márgenes suaves, sugiriendo estructuras menos profundas que $C$. rugosa.

$\mathrm{Si}$ bien algunos autores han extendido cuestionablemente su rango estratigráfico (e.g. Bradshaw, 1981), Cruziana furcifera ha sido documentada en sucesiones que se extienden desde el Tremadoc Superior hasta el Llandeilo (Crimes, 1975a). En las cuencas del noroeste y oeste de Argentina, C. furcifera es un componente típico de sucesiones arenigianasllanvirnianas (Aceñolaza, 1978; Mángano et al., 1996a; Mángano y Buatois, 1996). En sucesiones tremadocianas, C. furcifera y $C$. semiplicata pueden presentarse asociadas (Crimes, 1970a, 1975a). Crimes (1970a) y Crimes y Marcos (1976) señalaron un aumento en el tamaño de $C$. furcifera desde el Tremadoc Superior hasta el Arenig.

Cruziana furcifera es la icnoespecie que fue identificada por los trabajos paleontológicos previos 
realizados en la Formación Mojotoro (Borrello, 1966a; Aceñolaza, 1978; Volkheimer et al., 1980). Sin embargo, especímenes asignables a Cruziana furcifera sensu strictum sólo constituyen aproximadamente un $25 \%$ de la icnofauna. En la Formación Mojotoro, un número significativo de especímenes presentan relaciones transicionales entre $C$. furcifera y $C$. goldfussi (Fig. 3a) o C. furcifera y C. rugosa (Fig. 3b), lo cual indica que al menos una determinada especie de trilobites era capaz de producir las distintas icnoespecies y que éstas constituyen variedades etológicas. Cruziana furcifera ha sido reconocida en distintas unidades litoestratigráficas ordovícicas de Argentina. Mángano et al. (1996a) y Mángano y Buatois (1996) documentaron Cruziana furcifera en estratos arenigianos de la Formación Suri (Sistema del Famatina, provincia de Catamarca). Crossopodia scotica (Borrello, 1966b), de la Formación La Tinta, en las Sierras Septentrionales de la provincia de Buenos Aires, constituye otro registro de Cruziana furcifera en facies cuarcíticas (cf. Fillion y Pickerill, 1990: 26). Aceñolaza (1978) también mencionó la presencia de Cruziana furcifera en estratos arenigianos en la ladera occidental de la Sierra de Santa Bárbara.

\section{Cruziana goldfussi (Rouault, 1850)} Figs. 3a, 4

Material: siete ejemplares en las muestras números PIL 12258, PIL 10474 y PIL 14641, y siete adicionales observados en el campo.

\section{Descripción}

Crestas hipicnias bilobuladas rectas o formando la parte posterior de estructuras en cubeta. Marcas de rasguñaduras retroversas cubren la totalidad de los lóbulos. Marcas de rasguñaduras finas, de perfil anguloso, agrupadas en sets múltiples que presentan gran continuidad y tienden posteriormente a ponerse paralelos al surco medio. Crestas marginales prominentes, $1,7-3,7 \mathrm{~mm}$ de ancho, rara vez preservadas en ambos lados de la traza (Fig. 4b-c). El ancho de la traza varía entre 32,0 y 71,2 mm. La longitud máxima observada es de $220 \mathrm{~mm}$. Ángulo de la "V" de $30^{\circ}$ a $70^{\circ}$. Surco central recto y bien definido.

\section{Discusión}

Cruziana goldfussi es interpretada como una variedad etológica opistoclina del grupo rugosa, en la que habrían participado apéndices posteriores y de menor tamaño que los registrados en $C$. furcifera (cf. Seilacher, 1970). El arqueamiento del cuerpo producía el arrastre de las espinas pleurales o genales sobre el sedimento. Frecuentemente se considera que el rasgo diagnóstico de Cruziana goldfussi es la presencia de surcos marginales interpretados como producidos por espinas pleurales (Seilacher, 1970) o genales (Crimes, 1975c; Bergström, 1976). Bergström (1976) notó, sin embargo, que surcos marginales pueden estar presentes ocasionalmente en $C$. furcifera y C. rugosa (cf. Bergström, 1976: fig. 6). Esta observación ha sido confirmada en los ejemplares de
Cruziana de la Formación Mojotoro. La presencia de surcos marginales se genera por el arrastre pasivo de las espinas genales sobre el sedimento, y si bien su presencia puede ser determinada por la posición de excavación (i.e. opistoclina), su significación etológica es limitada. Al respecto, Bergström (1976) consideró que el modo de instrumentación de los apéndices debe constituir la icnotaxobase más significativa en la distinción de formas dentro del grupo rugosa. Dicho autor señala que la presencia o ausencia de marcas de espinas genales no constituye un buen carácter sistemático por distintas razones: (1) problemas de conservación relacionados con la profundidad e inclinación del céfalo, (2) trilobites filogenéticamente cercanos pueden diferir notablemente en esta característica y (3) existe un amplio rango de variaciones en el desarrollo de espinas genales en la ontogenia de numerosas especies de trilobites. Por otra parte, Cruziana goldfussi suele presentar marcas de rasguñaduras más finas que $C$. furcifera y $C$. rugosa, evidenciando el uso de apéndices de menor porte que aquellos utilizados en las otras variedades del grupo, y posiblemente posteriores (cf. Bergström, 1976; Durand, 1985).

Cruziana goldfussi está pobremente representada en la Formación Mojotoro, constituyendo aproximadamente el $15 \%$ de los ejemplares de Cruziana. El material analizado se caracteriza por la presencia de especímenes transicionales con otros miembros del grupo rugosa, comúnmente con forma de cubeta, en los que $C$. goldfussi se presenta invariablemente en la parte posterior de la estructura (cf. Seilacher, 1970: fig. 2). La otra forma de aparición de $C$. goldfussi es como estructuras bilobuladas rectas, en forma de cinta, de escasa convexidad y gran continuidad (Fig. 3a). En este último caso, la distinción entre $C$. furcifera y $C$. goldfussi es difícil de establecer. La presencia de marcas marginales unilaterales en el material analizado es interpretada como producto de una posición de excavación inclinada, no totalmente paralela al plano de estratificación (Fig. 4b-c). Al menos en dos muestras (PIL 10474 y PIL 14615) puede verificarse que estructuras acintadas asignables a $C$. goldfussi se disponen en niveles toponómicos más superficiales que C. rugosa.

\section{Cruziana rugosa d'Orbigny, 1842 Figs. 3b, 5}

Material: veintitrés ejemplares en las muestras número PIL 10473, PIL 10474, PIL 10475, PIL 10849, PIL 14608, PIL 14609, PIL 14610, PIL 14611, PIL 14612, PIL 14613, PIL 14614, PIL 14615, PIL 14617, PIL 14618, PIL 14619, PIL 14620, PIL 14627, PIL 14632, PIL 14633 y PIL 14634, y más de cuarenta ejemplares observados en el campo.

\section{Descripción}

Crestas hipicnias bilobuladas formando comúnmente la parte anterior o la totalidad de excavaciones cortas en forma de cubeta (Fig. 5b,d,f). Marcas de rasguñaduras angulosas y prominentes, agrupadas en sets de hasta 11 marcas. Los sets 
sucesivos no se superponen y están separados entre sí por corrugaciones transversas, aproximadamente perpendiculares al surco medio. El ancho de la traza varía entre 24,2 y 84,6 mm. La longitud máxima observada es de aproximadamente $170 \mathrm{~mm}$. En la mayoría de los especímenes analizados, la distancia entre corrugaciones es relativamente constante, si bien algunos ejemplares presentan distancias variables resultando en un aspecto más irregular (Fig. 5a,e). Numerosos especímenes presentan asimetría con relación al surco medio, mostrando las corrugaciones transversas sólo bien desarrolladas en uno de los lóbulos (Fig. 5e) o con relación al plano que divide la región anterior de la posterior, presentando las características típicas de $C$. rugosa en el tramo anterior de la estructura.

\section{Discusión}

Cruziana rugosa es la icnoespecie más abundante en la Formación Mojotoro, representando más del 60\% de la totalidad de los ejemplares de Cruziana presentes y aproximadamente el $90 \%$ de los especímenes de Cruziana en depósitos de planicies de marea. Cruziana rugosa corresponde a la variedad prosoclina del grupo rugosa (Seilacher, 1970, 1992). La producción de $C$. rugosa implicaría el accionar de un único par de apéndices anteriores, generando sets de marcas múltiples claramente individualizables, que no se superponen con sets sucesivos (Baldwin, 1977b). La peculiar morfología de Cruziana rugosa ha dado lugar a diversas interpretaciones en cuanto a los instrumentos utilizados en su producción. Bergström (1976) interpretó Cruziana rugosa como producida por las espinas lamelares de exitos, mientras que Seilacher $(1970,1992)$ consideró las múltiples marcas como producidas por pinzas o setas endopodales terminales multidigitadas. El rango estratigráfico de $C$. rugosa es Arenig-Llandeilo (Crimes, 1975a). Seilacher (1992) mencionó la presencia de representantes del grupo rugosa en el Ordovícico Superior de Bolivia, lo cual plantearía una extensión del rango del grupo hasta el Caradoc.

En la Formación Mojotoro, numerosos ejemplares de
C. rugosa presentan una configuración asimétrica con respecto al surco medio, producto de un proceso de excavación inclinada lateralmente (Fig. 5e). Por otra parte, Cruziana rugosa presenta un amplio rango de tamaños, sugiriendo que más de una especie de trilobites estuvo involucrada en su producción o la participación de organismos en distintos estadios ontogénicos. La casi totalidad de los ejemplares de Cruziana rugosa de la Formación Mojotoro corresponde a formas acintadas de escasa longitud, referidas como surcos en cubeta (bathtub furrows sensu Seilacher, 1992). El plano de estratificación ilustrado por Borrello (1966a) en la quebrada del Gallinato está cubierto en su casi totalidad por $C$. rugosa en forma de surcos en cubeta.

\section{Icnogénero Dimorphichnus Seilacher, 1955}

\section{Dimorphichnus isp.} Fig. 6b

Material: muestras número PIL 14624, PIL 14625, PIL 14627 y PIL 14628.

\section{Descripción}

Marcas de rasguñaduras asimétricas, preservadas como crestas hipicnias. Se distinguen claramente dos tipos de marcas de rasguñaduras: aquellas cortas constituidas por 4 a 7 crestas rectas subparalelas y otras elongadas, con forma sigmoidal, lisas o surcadas por 4 a 8 crestas que tienden a acompañar la forma de sigmoide de la rasguñadura. En la muestra PIL 14628, estas marcas están organizadas en series subparalelas asimétricas. Sin embargo, en el resto del material bajo estudio las marcas dimórficas se presentan sobrepuestas formando densas superficies indiferenciadas.

\section{Discusión}

Seilacher (1955) definió Dimorphichnus para englobar huellas asimétricas formadas por dos tipos de impresiones, unas elongadas y finas y otras cortas y

Figura 5. Cruziana rugosa. a. Ejemplar mostrando corrugaciones irregulares y asimetría con relación al surco medio. PIL 14620. b. Imbricación de distintos especímenes profundos en forma de cubeta en "U". Nótese la presencia de Arenicolites (cuadrante inferior derecho) cortando un ejemplar pequeño de C. rugosa. PIL 14621. c. Traza vermiforme (?Arenicolites) cortando de modo oblicuo al surco medio de C. rugosa. PIL 14609. d. Superficie mostrando superposición de especímenes de Cruziana rugosa emplazados a distinta profundidad. Nótese el espécimen central profundo mostrando la típica morfología en forma de "U". PIL 14634. e. C. rugosa excavada oblicuamente mostrando marcada asimetría con relación al surco medio. Nótese el desarrollo de prominentes corrugaciones transversales en el lóbulo de la derecha y la ausencia de éstas en el lóbulo de la izquierda. Skolithos linearis se dispone cortando a C. rugosa. PIL 14638. f. Vista en sección de un ejemplar de C. rugosa mostrando la morfología de las excavaciones en forma de cubeta en "V". PIL 14620. Barra = $1 \mathrm{~cm}$.

Cruziana rugosa. a. Specimen showing irregular corrugations and asymmetry with respect to the median furrow. PIL 14620. b. Imbrication of various deep, bathtube, U-shaped specimens. Note Arenicolites (lower right quadrangle) crosscutting a small specimen of C. rugosa. PIL 14621. c. Vermiform trace (?Arenicolites) obliquely crosscutting the median furrow of C. rugosa. PIL 14609. d. Surface displaying superposition of specimens of Cruziana rugosa emplaced at variable depths. Note central, deep specimen with typical U-shaped morphology. PIL 14634. e. C. rugosa showing marked asymmetry with respect to the median furrow. Prominent transverse corrugations occur only on lobe on the right. Skolithos linearis crosscuts C. rugosa. PIL 14638. f. Cross-sectional view of a specimen of C. rugosa showing bathtube, V-shaped morphology. PIL 14620. Bar $=1 \mathrm{~cm}$. 


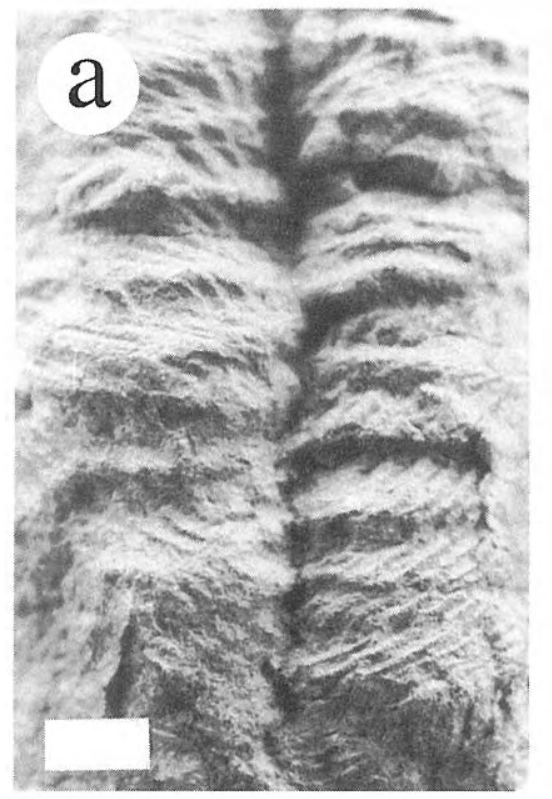

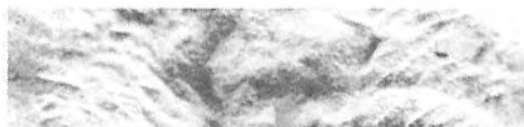

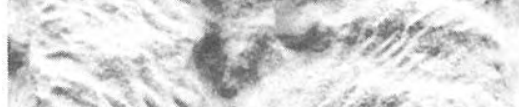

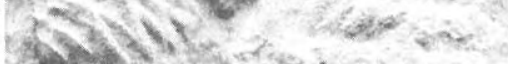

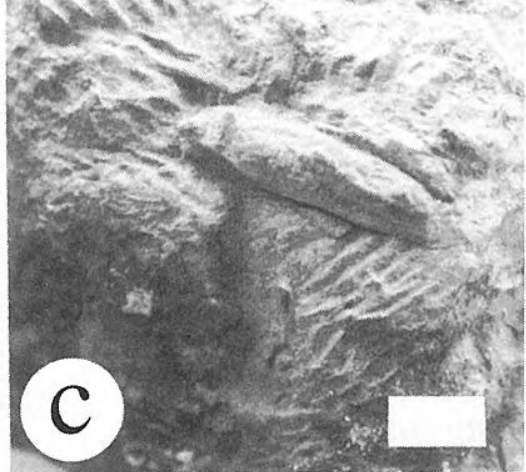

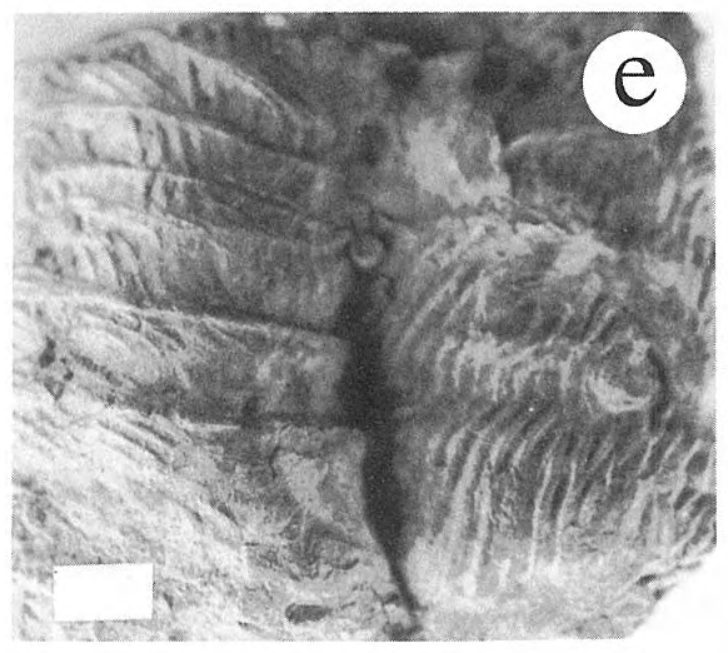

b
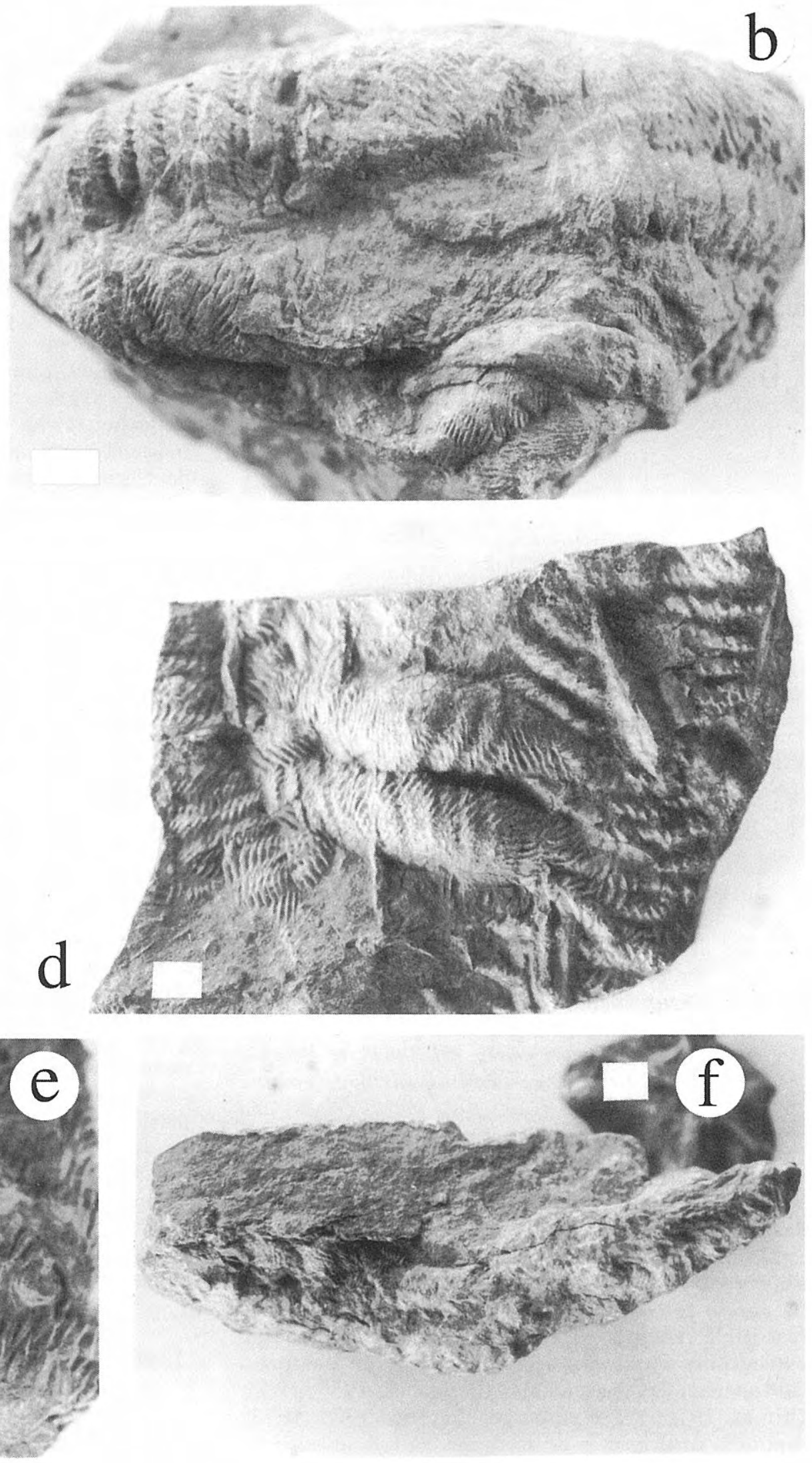

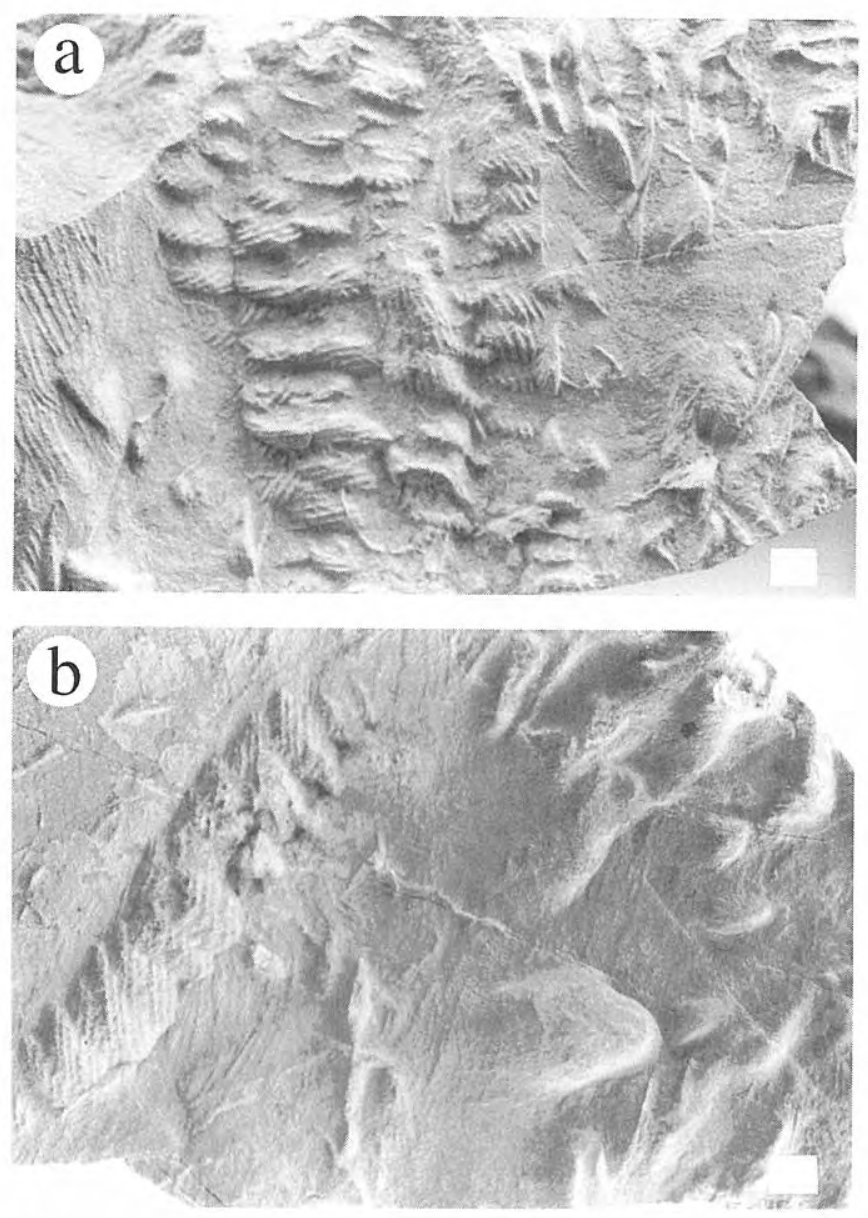

Figura 6. Vista basal de una capa con Dimorphichnus isp. a. Densa superposición de marcas de empuje prominentes y marcas de rastrillado sigmoidales con pobre organización. PIL 14628. b. Detalle de la misma superficie mostrando evidencias de erosión: turboglifos (derecha) y preservación parcial de un ejemplar de C. rugosa (lóbulo izquierdo). PIL 14627. Barra $=1 \mathrm{~cm}$.

Basal view of sandstone bed displaying Dimorphichnus isp. a. Dense superposition of prominent push marks and poorly organized, sigmoidal raking marks. PIL 14628. b. Detailed view of the same bedding surface showing evidence of erosion: flute marks (right) and partial preservation of a specimen of $\mathrm{C}$. rugosa (left lobe). PIL 14627. Bar $=1 \mathrm{~cm}$.

despuntadas. Seilacher (1955, 1990) interpretó estas estructuras como generadas por el movimiento oblicuo de trilobites, produciendo series alternantes de marcas cortas de apoyo (pushing marks) y marcas elongadas de rastrillaje (raking marks). Consecuentemente, estas estructuras corresponderían a trazas de pastoreo subsuperficiales (pascichnia) (Seilacher, 1955, 1990; Crimes, 1970b). Sin embargo, Osgood (1970, 1975) sugirió la participación de corrientes en la formación de Dimorphichnus, notando que la hipótesis del pastoreo indicaría una estrategia sumamente ineficiente de alimentación.
A diferencia del material asignado al icnogénero Cruziana (presente en más de treinta niveles estratigráficos a lo largo de la Formación Mojotoro), los especímenes asignables a Dimorphichnus muestran una distribución mucho más localizada. La clara asimetría observada entre las series de rasguñaduras permite distinguir este material de Monomorphichnus y Diplichnites. Una discusión más extensa acerca de Dimorphichnus y sus relaciones con Diplichnites, Monomorphichnus y Petalichnus puede encontrarse en Fillion y Pickerill (1990). A excepción de la muestra PIL 14628, el material en estudio asignado a Dimorphichnus es fragmentario, estando constituido mayormente por marcas dimórficas no claramente organizadas en series. Este modo de preservación es característico de Dimorphichnus y es el resultado de procesos de índole tafonómica, tales como preservación parcial de las marcas y gran superposición de especímenes (cf. Seilacher, 1990, p. 655). Dimorphichnus se presenta frecuentemente como subtrazas (undertraces) en la base de delgadas areniscas (Seilacher, 1990). Sin embargo, en el material estudiado, Dimorphichnus se encuentra en la base de capas relativamente potentes, de aproximadamente $10 \mathrm{~cm}$ de espesor, de areniscas con estratificación entrecruzada planar, lo cual podría indicar la mediación de un evento de exhumación, posiblemente vinculado a la migración de ondas submareales. La muestra PIL 14627 presenta pequeños turboglifos, indicando la acción de corrientes unidireccionales erosivas durante la sedimentación de la arenisca suprayacente (Fig. 6b). Estas corrientes fueron posiblemente responsables de la exhumación y preservación parcial de especímenes de Cruziana y Dimorphichnus (Fig. 6b). Por otra parte, Dimorphichnus en la Formación Mojotoro esta asociado a facies cuarcíticas submareales de escaso contenido en detritos orgánicos, lo cual hace adaptativamente ineficiente la clásica interpretación de Dimorphichnus como una estructura de pastoreo (cf. Osgood, 1975).

La morfología de las marcas de rasguñaduras multidigitadas no deja dudas acerca de la afinidad de Dimorphichnus isp. de la Formación Mojotoro con el grupo rugosa. El hecho de que el número de dígitos representados sea menor que en Cruziana rugosa tendría un origen tafonómico. En la muestra PIL 14628 también puede observarse una relación transicional entre marcas de apoyo de Dimorphichnus, que se vuelven más próximas entre sí hasta pasar a constituir una estructura comparable a Cruziana rugosa (Fig. 6a). Bergström (1976: fig. 19) ilustró marcas de rasguñaduras superficiales asignables conjuntamente a Dimorphichnus, comparables a las aquí analizadas.

\section{IMPLICANCIAS PALEOECOLÓGICAS Y PALEOAMBIENTALES}

El grupo rugosa presenta algunas características notables en cuanto a su modo de aparición y asociación facial. Numerosos autores han documentado la presencia de $C$. furcifera, $C$. goldfussi y C. rugosa en facies 
cuarcíticas vinculadas a ambientes mareales (e.g. Crimes y Marcos, 1976; Baldwin, 1977b; Durand, 1985; Fillion y Pickerill, 1990). En estas sucesiones, los integrantes del grupo rugosa se presentan de manera gregaria, comúnmente formando densas superficies pauciespecíficas. La distribución de trazas fósiles en las parasecuencias de la Formación Mojotoro muestra que las superficies portadores de Cruziana son más abundantes en las capas de arenisca fina de las facies heterolíticas de la planicie mixta, si bien también están localmente presentes en facies cuarcíticas de energía moderada a alta de la planicie arenosa y de zonas submareales de interbarra. Esta distribución se ve probablemente favorecida por el mayor potencial de preservación de las trazas de trilobites en facies heterolíticas. En sus estudios en La Serie de Los Cabos, España, Baldwin (1977b) notó que el $70 \%$ de los especímenes de Cruziana del grupo rugosa se encontraba en facies de línea de costa, particularmente en ambientes de playa y planicie intermareal. En la Formación Mojotoro, un punto de interés es la presencia de superficies densamente ocupadas por Cruziana rugosa en facies heterolíticas de la planicie intermareal, en particular de la planicie mixta y arenosa, donde constituye aproximadamente el $90 \%$ de los ejemplares de Cruziana allí presentes. Los parámetros ecológicos que gobiernan estos ambientes se apartan de las condiciones imperantes en ambientes marinos abiertos. La planicie intermareal representa condiciones límites para el rango de distribución de la gran mayoría de las especies marinas; sólo algunas especies encuentran posible y ventajoso incursionar en estos ambientes. A su vez, la planicie mareal integra la zona ecotonal entre el continente y el mar, donde numerosas especies anidan o incursionan en busca de alimento (Reise, 1985; Raffaelli y Hawkins, 1996; Mángano y Buatois, 1998). Fenton y Fenton (1937) interpretaron Rusophycus jenningsi como excavaciones producidas para albergar huevos y compararon el comportamiento de los trilobites con el del xifosúrido actual Limulus. Basándose en estudios recientes sobre etología de xifosúridos, Mángano et al. (1996b) compararon más detalladamente el comportamiento reproductivo de estos organismos y las estructuras de trilobites en facies de planicies mareales de la Formación Santa Rosita, e interpretaron la presencia de agrupamientos de Rusophycus profundos como posibles estructuras de nidada. La ausencia de estructuras rusofisiformes, de tipo estacionario, en las típicas asociaciones del grupo rugosa hace poco probable la hipótesis de estructuras de nidada. Otro móvil que atrae a numerosas especies marinas y terrestres a la planicie de marea es la búsqueda de alimento. Numerosas especies marinas están reguladas por el ciclo mareal (Palmer, 1995). La marea alta transporta larvas e individuos juveniles y adultos de diversas especies hacia la planicie, que pueden anidar en la zona intermareal o incursionar en busca de alimento y regresar a regiones submareales cuando la marea se retira. En ambientes actuales los principales visitantes adultos que ingresan a la planicie desde el hábitat marino son predadores, en particular peces y crustáceos (Little, 2000). Otras especies adoptan la infaunalización para mitigar las estresantes condiciones vinculadas con la exposición subaérea y, al bajar la marea, producen excavaciones en busca de protección, donde esperan la llegada de la nueva marea. Las superficies con especímenes de Cruziana rugosa en los depósitos de planicies mareales de la Formación Mojotoro registran muy posiblemente estructuras vinculadas a una estrategia de alimentación y no simples trazas de locomoción. Bergström (1976) interpretó Cruziana rugosa como una estructura de pastoreo (pascichnia) basándose en el hecho de que la energía empleada en la técnica de excavación de $C$. rugosa no era compatible con una estructura de simple locomoción (repichnia). Teniendo en cuenta esta interpretación, las estructuras del grupo rugosa registrarían las actividades de depositívoros móviles (sensu Bromley, 1996) que explotaban los depósitos con alto contenido de materia orgánica de la zona intermareal. En forma análoga a organismos marinos actuales, las especies de trilobites involucradas posiblemente incursionaban en los ambientes de planicie, valiéndose de la dinámica de las mareas. Por otra parte, la morfología de estructuras en cubeta que caracteriza a $C$. rugosa no parece responder al patrón morfológico de una clásica estructura de pastoreo. La morfología de Cruziana rugosa (i.e. estructuras cortas en forma de "U", relativamente profundas) y su asociación con estructuras vermiformes podría indicar estructuras de predación, en las cuales el predador se zambulle en el sedimento en busca de su presa. Esta hipótesis alternativa sería sustentada por evidencias paleobiológicas (ver "Implicancias Bioestratigráficas y Paleobiológicas"). Sin embargo, las observaciones icnológicas sobre relaciones con las estructuras vermiformes asociadas no son concluyentes. En primer lugar, y en relación con las estructuras verticales (Skolithos, Arenicolites), éstas cortan comúnmente a los ejemplares de Cruziana (Fig.3a,c; Fig. 4b-e). Por otra parte, en los casos de asociación con estructuras vermiformes horizontales (Palaeophycus), no se siguen los criterios propuestos para la identificación de estructuras de predación, tales como alineamiento de los ejes de ambas estructuras, contacto de la traza vermiforme con un sólo lóbulo y correlación de tamaños (cf. Bergström, 1973; Jensen, 1990; Brandt et al., 1995).

Por su parte, en las zonas submareales de ondas de arena de ambientes actuales, particularmente en los sectores de migración activa de formas de lecho, son pocas las especies adaptadas a sobrevivir (Wilson, 1982, 1986). La diversidad faunística aumenta hacia las zonas de ondas de arena de menores dimensiones y hacia los sectores más externos del complejo, donde las ondas de arena son reemplazadas por óndulas intercaladas con fango. La distribución de trazas fósiles en la Formación Mojotoro es consistente con este patrón de distribución faunística. Las capas de cuarcitas más potentes, correspondientes al núcleo de las barras, representan las zonas más activas del complejo y carecen de bioturbación. En las capas más delgadas se presentan asociaciones monoespecíficas de Skolithos linearis, 
representando superficies de colonización asociadas a pausas en la migración de las formas de lecho. Esto es coherente con observaciones en ambientes actuales que demuestran que los organismos suspensívoros son dominantes en zonas arenosas de alta energía de mareas (Wilson, 1982). Las superficies portadoras de trazas de trilobites se presentan en la base de delgadas capas de cuarcitas (que sólo excepcionalmente alcanzan espesores cercanos a los $10 \mathrm{~cm}$ ) asociadas a láminas pelíticas, acumuladas en sectores más externos del complejo de ondas de arena y zonas de interbarra. En complejos de ondas submareales actuales se ha observado un aumento en la proporción de organismos detritívoros y depositívoros concomitante con un aumento del contenido de sedimento fino. Wilson (1982) notó un predominio de organismos epifaunales en zonas de erosión intensa, pero el potencial de conservación de las estructuras producidas por dichos organismos en tales sectores es prácticamente nulo.

La asociación de trazas de trilobites de la Formación Mojotoro descripta en este trabajo es asignable a la icnofacies de Cruziana, si bien la icnodiversidad en asociaciones de ambientes marginales es comúnmente moderada a baja. En la Formación Mojotoro, los especímenes de Cruziana del grupo rugosa constituyen aproximadamente un $80 \%$ de las trazas presentes en la icnocenosis correspondiente a las facies heterolíticas de tipo 1. Los otros componentes de la icnocenosis corresponden a pequeños especímenes de Cruziana pobremente preservados y estructuras vermiformes de tipo Palaeophycus. Superpuestos a esta icnocenosis, de orientación predominantemente horizontal, se disponen elementos verticales de la icnofacies de Skolithos, vinculados al ingreso súbito de arenas. Mángano et al. (1996b) documentaron fábricas palimpsésticas semejantes en el tramo inferior de la Formación Santa Rosita (Cámbrico Superior-Tremadoc).

Mángano y Buatois (1999) enfatizaron el gradiente invertido de icnofacies que se registra en costas mareales. En ambientes dominados por procesos mareales, la energía se incrementa desde la planicie fangosa hacia la zona submareal, resultando en ambientes protegidos de menor energía sobre la costa y ambientes más energéticos costa afuera. La proporción de sedimento arenoso, cantidad de nutrientes en suspensión, tasa de sedimentación, grado de movilidad de las formas de lecho y contenido de oxígeno aumenta concomitantemente con la energía hacia los sectores submareales, en forma inversa a lo que sucede en costas dominadas por oleaje. Consecuentemente, en costas mareales, la icnofacies de Skolithos tiende a ubicarse hacia la zona submareal y la icnofacies de Cruziana hacia la zona intermareal, particularmente en la planicie arenosa y planicie mixta. La presencia de la icnofacies de Cruziana en planicies mareales es consistente con observaciones en ambientes actuales (e.g. Bajard, 1966; Swinbanks y Murray, 1981; Frey et al., 1987). Al igual que sucede en ambientes dominados por oleaje, las variaciones en los parámetros locales pueden generar variaciones en este gradiente. En ambientes dominados por olas, la acción de tormentas permite la colonización de capas arenosas por elementos de la icnofacies de Skolithos, posibilitando el desarrollo de esta asociación en un ambiente donde la icnofacies de Cruziana representa a la fauna residente (Pemberton y Frey, 1984). Del mismo modo, elementos de la icnofacies de Cruziana pueden presentarse en los sectores localizados de interbarra, que actúan como refugios de las condiciones extremas imperantes en la zona submareal en costas dominadas por la acción de mareas, y la icnofacies de Skolithos puede presentarse en sectores intermareales, relacionada con el desarrollo de canales y formas de lecho de moderada a alta intensidad de corrientes (Mángano et al., 1996b; Mángano y Buatois, 1999).

\section{IMPLICANCIAS BIOESTRATIGRÁFICAS Y PALEOBIOLÓGICAS}

Distintos autores han reconocido la importancia de Cruziana en zonaciones estratigráficas, particularmente en estratos "no fosilíferos" del Paleozoico Inferior. Crimes (1968) distinguió estratos tremadocianos de arenigianos sobre la base de icnoespecies de Cruziana. Una icnoestratigrafía de Cruziana también permitió realizar una zonación del Cámbrico Superior-Tremadoc en el Norte de Gales (e.g. Crimes, 1969, 1970b, 1975b), del Cámbrico Superior-Tremadoc-Arenig del norte y oeste de España (e.g. Arboleya, 1973; Moreno et al., 1976; Crimes y Marcos, 1976; Baldwin, 1977b; Pickerill et al., 1984; Gámez-Vintaned, 1995), del este de Terranova (e.g. Bergström, 1976; Pickerill y Fillion, 1983; Fillion y Pickerill, 1990) y del norte y este de Africa (e.g. Crimes, 1981; Alidou et al., 1986; Seilacher, 1990, 1992). La icnoestratigrafía de Cruziana postula que las trazas producidas por la actividad excavadora de trilobites pueden utilizarse como icnofósiles con valor bioestratigráfico ya que estas estructuras registran rasgos morfológicos finos (e.g. morfología de los apéndices, marcas de espinas genales) de las especies productoras. Como las especies de trilobites presentan estrechos rangos estratigráficos, consecuentemente también lo harán las estructuras producidas por dichas especies. Cabe consignar que no existe necesariamente una correlación uno a uno entre una dada icnoespecie de Cruziana y una especie de trilobites, sino que un grupo de especies, posiblemente vinculadas filogenéticamente, pudo ser responsable de la producción de determinada icnoespecie. Seilacher $(1970,1992,1994)$ desarrolló ampliamente los fundamentos de la denominada "icnoestratigrafía de Cruziana", reconociendo más de 30 icnoespecies de Cruziana (y Rusophycus) con valor bioestratigráfico debido a su rango temporal restringido. A su vez, las icnoespecies integran "grupos" que pueden tener una única aparición estratigráfica (e.g. grupo semiplicata, Cámbrico Superior-Tremadoc) o presentarse en más de un intervalo estratigráfico (e.g. grupo omanica, Cámbrico Superior-Tremadoc, Caradoc y Silúrico con sus respectivas especies). La identificación de icnoespecies y su integración en grupos se basa en la 
morfología y tipo de agrupamientos de las marcas de rasguñaduras, presencia/ausencia de marcas exopodales, impresiones genales, etc. Un punto muy importante es el reconocimiento de la morfología de marcas endopodales (claw formula o digit mark), que permite reconstruir las características de los apéndices productores.

Las trazas del grupo rugosa constituyen, junto con las del grupo semiplicata, las mejor conocidas y más utilizadas en bioestratigrafía. El icnogénero Cruziana fue originalmente establecido sobre la base de material correspondiente a $C$. rugosa y $C$. furcifera (d'Orbigny, 1842). Por otra parte, este grupo presenta amplia distribución paleogeográfica en las plataformas del Ordovícico temprano a medio periféricas de Gondwana (Seilacher, 1970, 1992). Los especímenes de Cruziana del grupo rugosa se caracterizan por sets de hasta doce marcas de rasguñaduras, de perfil anguloso, con ausencia de marcas exopodales, si bien pueden estar presentes surcos externos (Seilacher, 1970, 1990). Algunos integrantes del grupo rugosa, en particular Cruziana furcifera, han sido mencionados en el Tremadoc superior, estas formas son comúnmente de menor tamaño y se encuentran frecuentemente acompañadas de típicas icnoespecies tremadocianas (e.g. Cruziana semiplicata), en ausencia de $C$. rugosa. Cruziana rugosa parece no estar presente en estratos más antiguos que el Arenig (Crimes, 1975a). Recientemente, Magwood y Pemberton (1990) desafiaron el paradigma de la icnoestratigrafía de Cruziana documentando la aparente presencia de Cruziana furcifera, C. goldfussi y C. rugosa en rocas del Cámbrico Inferior de Canadá. El análisis morfoetológico detallado de estas estructuras demuestra que, dejando de lado algunas similitudes, los especímenes cámbricos corresponderían a una nueva icnoespecie, Cruziana pectinata, presentando significativas diferencias morfológicas y construccionales con el grupo rugosa (cf. Seilacher, 1994). Por otra parte, el límite superior del rango de $C$. rugosa no se encuentra aún bien establecido. Seilacher (1992, p. 1572) explicó la excepcional presencia de especímenes de Cruziana asignables al grupo rugosa en el Caradoc de Bolivia como vinculada a la persistencia local de facies de tipo Grès Armoricain.

Basándose en evidencias biogeográficas, estratigráficas y morfológicas, Bergström (1973, 1976) concluyó que $C$. rugosa, $C$. furcifera y $C$. goldfussi registraban las actividades de excavación de trilobites de la Superfamilia Asaphacea (sensu Bergström, 1973). Otros autores (e.g. Seilacher, 1962, 1990) han propuesto a Illaenus como organismo productor de C. rugosa y formas relacionadas. Sin embargo, de acuerdo a Bergström (1973, p. 58), Illaenus no habría podido excavar eficientemente en posición prosoclina, lo cual permite al menos descartarlo como productor de $C$. rugosa. Por otra parte, Illaenus y numerosas especies de illaénidos habrían habitado ambientes arrecifales, en los cuales el modo de vida reflejado por los representantes del grupo rugosa sería, ciertamente, poco esperable. En relación con la icnofauna de la Formación Mojotoro y otros registros del grupo rugosa en el noroeste y oeste de Argentina, las evidencias bioestratigráficas y estratigráficas permiten descartar a Illaenus como productor del grupo rugosa y señalan a los asáfidos como los productores más plausibles. En primer lugar, Illaenus y la familia Illaenidae se encuentran ausentes en la Cordillera Oriental (cf. Waisfeld et al., 1999), donde las estructuras del grupo rugosa se encuentran ampliamente representadas en ambientes marinos someros, particularmente en el intervalo Arenig inferior-Llanvirn. Este rango estratigráfico coincide con el florecimiento de sucesivas faunas de asáfidos. Waisfeld et al. (1999) señalaron que para el Arenig inferior, el 56\% de la fauna de trilobites corresponde a asáfidos, mientras que este grupo constituiría aproximadamente el $25 \%$ de la trilobitofauna en el Arenig medio. En numerosos casos, coquinas con abundantes restos de asáfidos se encuentran asociadas a representantes del grupo rugosa en sucesiones de plataforma interna (Mángano y Buatois, 1996). Finalmente, el rango de tamaño de los especímenes de Cruziana del grupo rugosa es compatible con el rango de tamaño de la fauna de asáfidos. En un reciente trabajo sobre los hábitos alimenticios de los trilobites, Fortey y Owens (1999) vinculan a los asáfidos con hábitos tróficos predatorios. Estos autores ilustran un ejemplar de Rusophycus asociado a Ogygynus armoricanus proveniente de estratos arenigianos de la Grès Armoricain. Además de las evidencias morfológicas (e.g. morfología del hipostoma, anatomía de los podómeros basales), estos autores señalan el tamaño como un importante indicador de formas predadoras. Al respecto cabe destacar que los representantes del grupo rugosa se caracterizan por rangos de tamaño que sugieren productores de gran porte. Las trazas de predación de trilobites han sido tradicionalmente asociadas a excavaciones de tipo estacionario (i.e. Rusophycus); aparentemente, las formas predadoras estarían equipadas de un sistema sensor que les permitiría detectar vibraciones y localizar eficientemente a la presa en el sedimento subyacente (Jensen, 1990; Fortey y Owens, 1999). En los casos documentados, las presas consistían en vermes que habitaban estructuras subhorizontales a inclinadas. El ejemplo paradigmático, Rusophycus dispar, permite reconstruir pormenorizados detalles acerca de la estrategia de cacería implementada (cf. Jensen, 1990). En contraposición, el grupo rugosa está constituido dominantemente por formas acintadas de considerable longitud que sugieren depositívoros móviles. Algunas superficies densamente cubiertas con C. furcifera y $C$. goldfussi se asemejan en su modo de aparición a las densas superficies cubiertas por $C$. semiplicata, ésta última interpretada clásicamente como una estructura de pastoreo (Seilacher, 1970; Fortey y Owens, 1999). Sin embargo, las cortas estructuras en forma de cubeta que caracterizan a $C$. rugosa, no parecen en principio compatibles con una estrategia de tipo pascichnia. El hecho de que $C$. rugosa sea dominante en facies de planicie mareal de la Formación Mojotoro, donde las formas continuas de tipo acintado son raras, sugiere que esta icnoespecie podría estar relacionada con una estrategia de predación. Como fue previamente señalado, la icnofauna de la formación Mojotoro está 
dominada por C. rugosa, existiendo un número significativo de formas transicionales. De esto puede inferirse que el productor de C. rugosa era capaz de generar tambien C. furcifera y C. goldfussi. Sin embargo, esto no significa que otras especies de trilobites no pudieran producir formas acintadas de $C$. furcifera y $C$. goldfussi, sin producir C. rugosa. De hecho, como fue señalado, el rango de $C$. rugosa no es exactamente coincidente con el de $C$. furcifera y $C$. goldfussi. Por otra parte, mientras las formas acintadas presentan un amplio rango de distribución ambiental desde plataforma hasta ambientes litorales, C. rugosa parece presentar una distribución más acotada, siendo netamente dominante en ambientes litorales, particularmente en planicies mareales y sectores submareales someros. Teniendo en cuenta la evidencia paleobiológica, y haciendo la salvedad de que las evidencias icnológicas no son concluyentes, el grupo rugosa pudo haber incluido como productores tanto a especies de depositívoros móviles como a predadores.

Resumiendo, la presencia del grupo rugosa en la Formación Mojotoro, sumada a la ausencia de $C$. semiplicata, indica una edad Arenig-Llandeilo para esta unidad, compatible con el control bioestratigráfico suministrado por el contenido fósil de las unidades infra y suprayacentes. El dominio de Cruziana rugosa es de más difícil evaluación ya que esta variedad etológica podría evidenciar un control de tipo ecológico (i.e. incursiones de algunas especies en busca de alimento a la planicie mareal) o tafonómico (preservación de estructuras más profundas). Distintas líneas de evidencia señalan a los asáfidos como los productores más probables del grupo rugosa.

Los extensos afloramientos del Cambro-Ordovícico del norte argentino y Bolivia abren un promisorio campo para comprobar las hipótesis paleoecológicas que surgen del estudio de las trazas de trilobites de la Formación Mojotoro y explorar la aplicabilidad del paradigma icnoestratigráfico de Cruziana.

\section{CONCLUSIONES}

1. La Formación Mojotoro del noroeste argentino alberga una abundante icnofauna de trilobites, principalmente en depósitos acumulados en las zonas externas de los complejos de ondas de arena, interbarras y planicies intermareales. El icnogénero Cruziana, representado por las icnoespecies del grupo rugosa $(C$. rugosa, C. furcifera y C. goldfussi), es la forma dominante, si bien se ha registrado también el icnogénero Dimorphichnus.

2. Los paquetes potentes de cuarcitas formados en las zonas más activas de los complejos de ondas de arena típicamente carecen de bioturbación. Las capas más delgadas, que representan sedimentación en ondas de arena más pequeñas y márgenes de barras, contienen asociaciones correspondientes a la icnofacies de Skolithos.

3. La alta densidad de trazas de trilobites en facies heterolíticas con estructuras indicativas de emersión y que se disponen en el tramo superior de las parasecuencias registraría las actividades de alimentación de trilobites (¿predatorias?) en planicies de mareas. Estas incursiones periódicas hacia la costa fueron probablemente controladas por los ciclos mareales.

4. La presencia del grupo rugosa y la ausencia de $C$. semiplicata indican una edad Arenig-Llandeilo para la Formación Mojotoro, lo cual es consistente con la edad sugerida por el contenido faunístico de las unidades infra y suprayacentes.

5. Se sugiere a los asáfidos como productores del grupo rugosa.

\section{AGRADECIMIENTOS}

Agradecemos a Julio Monteros, Fernando Muñiz y Eric Gomez-Hasselrot su colaboración en las tareas de campo. Rodolfo Aredes se encargó de la preparación de los ejemplares recolectados y Eric Gomez-Hasselrot de la confección de los dibujos. También agradecemos a Florencio Aceñolaza, quien durante la década de los 80 recolectó una significativa cantidad de especímenes en la quebrada de Gallinato. Hacemos extensivo nuestro agradecimiento a los árbitros de la revista, José Carlos García Ramos y José Antonio Gámez Vintaned. Mángano agradece el apoyo de la Fundación Antorchas, la Agencia Nacional de Promoción Científica, The Percy Sladen Memorial Fund y Sigma Delta Epsilon (Women in Science). Buatois agradece un subsidio otorgado por el Consejo de Investigaciones de la Universidad Nacional de Salta. Este trabajo es una contribución al Proyecto IGCP 410 "The Great Ordovician Biodiversification Event".

\section{BIBLIOGRAFÍA}

Aceñolaza, F.G. 1973. Sanbernardaspis pygantha nov. gen. et nov. sp. (Trilobita-Asaphidae) del cerro San Bernardo, Salta, República Argentina. Ameghiniana, 10, 132-138.

Aceñolaza, F.G. 1978. El Paleozoico inferior de Argentina según sus trazas fósiles. Ameghiniana, 15, 15-64.

Aceñolaza, F.G. y Durand, F.R. 1978. Trazas de trilobites en estratos del Ordovícico basal de la Puna Argentina. Acta Geológica Lilloana, 15, 5-12.

Aceñolaza, F.G. y Fernández, R. 1978. Trazas fósiles en el Ordovícico inferior de la Sierra de Cajas, Jujuy. Acta Geológica Lilloana (Supl.), 14, 33-37.

Albanesi, G. and Rao, R. 1996. Conodont fauna from the Santa Gertrudis Formation (Middle-Late Ordovician), Eastern Cordillera, Northwestern Argentina. Sixth International Conodont Symposium (ECOS VI) 1996, Warszawa, Abstracts, 3.

Alidou, S., Lang, J., Roman, I. et Seilacher, A. 1986. Elements de datation en faveur d`un age Paléozoique et Mésozoïque du bassin de Kandi (Nord-Est du Bénin, Afrique de l'Ouest). Journal of African Earth Sciences, 5, 339-344.

Anderton, R. 1976. Tidal-shelf sedimentation: an example from the Scottish Dalradian. Sedimentology, 23, 429-458. 
Arboleya, M.L. 1973. Nota sobre la icnofauna del Cámbrico de la costa asturiana entre Cudillero y Ballota (NW de España). Breviora Geológica Astúrica, 17, 37-42.

Astini, R.A. 1994. Interpretación estratigráfica de la Formación Sepulturas (Ordovícico Inferior) y unidades análogas del noroeste argentino: La Aloformación Sepulturas. Actas V Reunión Argentina de Sedimentología, San Miguel de Tucumán, 9-14.

Bajard, J. 1966. Figures et structures sédimentaires dans la zone intertidale de la partie orientale de la Baie du MontSaint-Michel. Revue de Géographie physique et de Géologie Dynamique, 8, 39-111.

Baldwin, C.T. 1977a. Internal structures of trilobite trace fossils indicative of an open surface furrow origin. Palaeogeography, Palaeoclimatology, Palaeoecology, 21, 273-284.

Baldwin, C.T. 1977b. The stratigraphy and facies associations of trace fossils in some Cambrian and Ordovician rocks of north western Spain. In: Trace Fossils 2 (Eds. T.P. Crimes and J.C. Harper). Geological Journal Special Issue, 9, 9-40.

Benedetto, J.L. 1999. El género Drabovinella (Brachiopoda) en el Caradociano de la sierra de Mojotoro, provincia de Salta, Argentina. Ameghiniana, 36, 235-238.

Bergström, J. 1973. Organization, life and systematics of trilobites. Fossils and Strata, 2, 1-69.

Bergström, J. 1976. Lower Palaeozoic trace fosils from eastern Newfoundland. Canadian Journal of Earth Sciences, 13, 1613-1633.

Berven, R.J. 1966. Cardium sandstone bodies, CrossfieldGarrington area, Alberta. Bulletin of Canadian Petroleum Geology, 14, 208-240.

Borrello, A. 1966a. Las facies de Cruziana en el Ordovícico del Norte Argentino. Ameghiniana, 4, 185-188.

Borrello, A. 1966b. Trazas, restos tubiformes y cuerpos fósiles problemáticos de la Formación La Tinta. Sierras Septentrionales - Provincia de Buenos Aires. Paleontografía Bonaerense, Fascículo V, Provincia de Buenos Aires, Gobernación, Comisión de Investigaciones Científicas, La Plata. 42 pp.

Bradshaw, M.A. 1981. Paleoenvironmental interpretations and systematics of Devonian trace fossils from the Taylor Group (lower Beacon Supergroup), Antarctica. New Zealand Journal of Geology and Geophysics, 24, 615-652.

Brandt, D.S., Meyer, D.L. and Lask, P.B. 1995. Isotelus (trilobita) "hunting burrow" from Upper Ordovician strata, Ohio. Journal of Paleontology, 69, 1079-1083.

Bridges, B.H. 1982. Ancient offshore tidal deposits. In: Offshore tidal sands: processes and deposits (Ed. A.H. Stride). Chapman and Hall, New York, 172-192.

Bristow, C.S. 1995a. Internal geometry of ancient tidal bedforms revealed using ground penetrating radar. In: Tidal signatures in modern and ancient sediments (Eds. B.W. Flemming and A. Bartholomä). Special Publication International Association of Sedimentologists, 24, 313-328.

Bristow, C.S. 1995b. Facies analysis in the Lower Greensand using ground-penetrating radar. Journal of the Geological Society, London, 152, 591-598.
Bromley, R.G. 1996. Trace Fossils. Biology, taphonomy and applications. Chapman \& Hall, London, 361 pp.

Bromley, R.G. and Frey, R.W. 1974. Redescription of the trace fossil Gyrolithes and taxonomic evaluation of Thalassinoides, Ophiomorpha and Spongeliomorpha. Bulletin of the Geological Society of Denmark, 23, 311 335.

Comisión Internacional de Nomenclatura Zoológica, 2000. Código Internacional de Nomenclatura Zoológica, $4^{\mathrm{a}}$ ed. Museo Nacional de Ciencias Naturales, Caja España, ICZN, Sociedad de Amigos del Museo Nacional de Ciencias Naturales, Madrid, 156 pp.

Crimes, T.P. 1968. Cruziana: a stratigraphically useful trace fossil. Geological Magazine, 105, 360-364.

Crimes, T.P. 1969. Trace fossils from the CambroOrdovician rocks of North Wales and their stratigraphic significance. Geological Journal, 6, 333-337.

Crimes, T.P. 1970a. The significance of trace fossils in sedimentology, stratigraphy and palaeoecology, with examples from Lower Palaeozoic strata. In: Trace Fossils (Eds. T.P. Crimes and J.C. Harper). Geological Journal Special Issue, 3, 101-126.

Crimes, T.P. 1970b. Trilobite tracks and other trace fosils from the Upper Cambrian of North Wales. Geological Journal, 7, 47-68.

Crimes, T.P. 1975a. The stratigraphical significance of trace fossils. In: The Study of Trace Fossils. A Synthesis of Principles, Problems, and Procedures in Ichnology (Ed. R.W. Frey). Springer-Verlag, New York, Heidelberg, Berlin, 109-130.

Crimes, T.P. 1975b. Trilobite traces from the Lower Tremadoc of Tortworth. Geological Magazine, 112, 3346.

Crimes, T.P. 1975c. The production and preservation of trilobite resting and furrowing traces. Lethaia, 8, 35-48.

Crimes, T.P. 1981. Lower Palaeozoic trace fossils of Africa. In: Lower Paleozoic of the Middle East, Eastern and Southern Africa, and Antarctica (Ed. C.H. Holland). John Wiley \& Sons Ltd., New York, 189-198.

Crimes, T.P. and Anderson, M.M.. 1985. Trace fossils from Late Precambrian-Early Cambrian strata of southeastern Newfoundland (Canada): temporal and environmental implications. Journal of Paleontology, 59, 310-343.

Crimes, T.P. and Marcos, A. 1976. Trilobite traces and the age of the lowest part of the Ordovician reference section for NW Spain. Geological Magazine, 113, 349-356.

Crimes, T.P., Legg, I., Marcos, A. and Arboleya, M. 1977. ?Late Precambrian - low Lower Cambrian trace fossils from Spain. In: Trace Fossils 2 (Eds. T.P. Crimes and J.C. Harper). Geological Journal Special Issue, 9 , 91-138.

Dalrymple, R.W. 1984. Morphology and internal structure of sandwaves in the Bay of Fundy. Sedimentology, 31, 365-382.

Dalrymple, R.W. 1992. Tidal depositional systems. In: Facies models: Response to sea level change (Eds. R.G. Walker and N.P. James). Geological Association of Canada, Toronto, 195-218.

Durand, J. 1985. Le Grès Armoricain. Sédimentologie-Traces fossiles. Milieux de dépôt. Mémoires et 
Documents, Centre Armoricain d'Etude Structurale des Socles, 3, 1-150.

El-Khayal, A.A. and Romano, M. 1988. A revision of the upper part of the Saq Formation and Hanadir Shale (lower Ordovician) of Saudi Arabia. Geological Magazine, 125, 161-174.

Fenton, C.L. and Fenton, M.A. 1937. Trilobite "nests" and feeding burrows. American Midland Naturalist, 18, 446-451.

Fillion, D. and Pickerill, R.K. 1990. Ichnology of the Lower Ordovician Bell Island and Wabana Groups of eastern Newfoundland. Palaeontographica Canadiana, 7, 1-119.

Fortey, R.A. and Owens, R.M. 1999. Feeding habits in trilobites. Palaeontology, 42, 429-465.

Frey, R.W. and Wheatcroft, R.A. 1989. Organism-substrate relations and their impact on sedimentary petrology. Journal of Geological Education, 37, 261-279.

Frey, R.W., Howard, J.D. and Hong, J.S. 1987. Prevalent Lebensspuren on a modern macrotidal flat, Inchon, Korea: Ethological and environmental significance. Palaios, 2, 571-593.

Gámez-Vintaned, J.A. 1995. Los materiales prehercínicos de la Sierra del Moncayo (Cadena Ibérica Oriental, España) y su contenido paleoicnológico. Boletín de la Real Sociedad Española de Historia Natural (Sección Geológica), 90, 21-50.

Goldring, R. and Seilacher, A. 1971. Limulid undertracks and their sedimentological implications. Neues Jahrbuch für Geologie und Paläontologie, Abhandlungen, 137, 422-442.

Harrington, H.J. 1938. Sobre las faunas del Ordovícico Inferior del norte argentino. Revista del Museo de La Plata (nueva serie), Sección Paleontología, 1, 109-189.

Harrington, H.J. 1957. Ordovician Formations of Argentina. In: Ordovician Trilobites of Argentina (Eds. H.J. Harrington and A.F. Leanza). The University of Kansas Special Publication, 1, 1-59.

Hein, F.J. 1987. Tidal/littoral offshore shelf deposits - Lower Cambrian Gog Group, southern Rocky Mountains, Canada. Sedimentary Geology, 52, 155-182.

Jensen, S. 1990. Predation by early Cambrian trilobites on infaunal worms - evidence from the Swedish Mickwitzia Sandstone. Lethaia, 23, 29-42.

Klein, G. de V. 1971. A sedimentary model for determining paleotidal range. Geological Society of America, Bulletin, 82, 2585-2592.

Klein, G. de V. 1977. Clastic Tidal Facies. CEPCO, Champaign, Illinois, $149 \mathrm{pp}$.

Kolb, S. and Wolf, R. 1979. Distribution of Cruziana in the Lower Ordovician sequence of Celtiberia (NE Spain) with a revision of the Cruziana rugosa-group. Neues Jahrbuch für Geologie und Paläontologie, Monatshefte, 1979, 457-484.

Little, C. 2000. The Biology of Soft Shores and Estuaries. Oxford University Press, New York, 252 pp.

Magwood, J.P. and Pemberton, S.G. 1990. Stratigraphic significance of Cruziana: new data concerning the Cambrian-Ordovician ichnostratigraphic paradigm. Geology, 18, 729-732.

Mángano, M.G. and Buatois, L.A. 1996. Shallow marine event sedimentation in a volcanic arc-related setting: the Ordovician Suri Formation, Famatina Range, northwest Argentina (Famatina System). Sedimentary Geology, 105, 63-90.

Mángano, M.G. y Buatois, L.A. 1997. Análisis icnológico comparativo de planicies mareales carboníferas del este de Kansas. Memorias 1er Congreso Latinoamericano de Sedimentología, Isla Margarita, 2, 1-6.

Mángano, M.G. y Buatois, L.A. 1998. Icnofacies de costas mareales y su importancia en reconstrucciones paleoambientales de sucesiones marinas someras. $7^{a}$ Reunión Argentina de Sedimentología, Salta, 1, 158-159.

Mángano, M.G. and Buatois, L.A. 1999. Ichnofacies models in Early Paleozoic tide-dominated quartzites: onshoreoffshore gradients and the classic Seilacherian paradigm. Acta Universitatis Carolinae, 43, 151-154.

Mángano, M.G., Buatois, L.A. y Aceñolaza, F.G. 1996a. Icnología de ambientes marinos afectados por vulcanismo: La Formación Suri, Ordovícico del extremo norte de la sierra de Narváez, Sistema del Famatina. Asociación Paleontológica Argentina, Publicación Especial, 4, 69-88.

Mángano, M.G., Buatois, L.A. and Aceñolaza, G.F. 1996b. Trace fossils and sedimentary facies from an Early Ordovician tide-dominated shelf (Santa Rosita Formation, northwest Argentina): Implications for ichnofacies models of shallow marine successions. Ichnos, 5, 53-88.

Mángano, M.G., Buatois, L.A. y Moya, M.C. 1999. Icnología de una sucesión marina somera dominada por mareas en el Paleozoico inferior del noroeste argentino, Formación Mojotoro, Ordovícico de Salta. $14^{\circ}$ Congreso Geológico Argentino, Actas, 1, 49-50.

Martinsson, A. 1965. Aspects of a Middle Cambrian thanatotope on Öland. Geologiska Föreningens $i$ Stockholm Förhandlingar, 87, 181-230.

McCave, I.N. 1971. Sand waves in the North Sea off the coast of Holland. Marine Geology, 10, 199-225.

Monaldi, C.R. 1982. Reasignación genérica de Calymenella? zaplensis, Harrington y Leanza, 1957 (Trilobita). Revista de la Asociación Geológica Argentina, 37, 261-267.

Monaldi, C.R. y Monaldi, O.H. 1978. Hallazgo de una fauna en la Formación Santa Gertrudis (Ordovícico), provincia de Salta, República Argentina. Revista de la Asociación Geológica Argentina, 33, 245-246.

Moreno, F., Vegas, R. y Marcos, A. 1976. Sobre la edad de las series ordovícicas y cámbricas relacionadas con la discordancia "sárdica" en el anticlinal de Valdelacasa (Montes de Toledo, España). Breviora Geológica Astúrica, 20, 8-16.

Moya, M.C. 1998. El Paleozoico inferior en la sierra de Mojotoro, Salta - Jujuy. Revista de la Asociación Geológica Argentina, 53, 219-238.

Moya, M.C., Malanca, S., Monteros, J.A. y Cuerda, A. 1994. Bioestratigrafía del Ordovícico Inferior en la Cordillera Oriental argentina basada en graptolitos. Revista Española de Paleontología, 9, 91-104.

Orbigny, A. d'. 1842. Voyage dans l'Amérique méridionale (le Brésil, la République orientale de l'Uruguay, la République Argentine, la Patagonie, la République du 
Chili, la République de Bolivia, la République du Pérou) exécuté pendant les années 1826, 1827, 1828, 1829, 1830, 1831, 1832 et 1833. v. 3, pt. 4 (Paléontololgie). Pitois-Leverault, Paris. Leverault, Strasbour, 188 pp.

OrXowski, S., Radwański, A. and Roniewicz, P. 1971. Ichnospecific variability of the Upper Cambrian Rusophycus from the Holy Cross Mountains. Acta Geologica Polonica, 21, 341-348.

Osgood, R.G., Jr. 1970. Trace fossils of the Cincinnati area. Palaeontographica Americana, 6, 277-444.

Osgood, R.G., Jr. 1975. The Paleontological Significance of Trace Fossils. In: The Study of Trace Fossils. A Synthesis of Principles, Problems, and Procedures in Ichnology (Ed. R.W. Frey). Springer-Verlag, New York, Heidelberg, Berlin, 87-108.

Palmer, J.D. 1995. The Biological Rhythms and Clocks of Intertidal Animals. Oxford University Press, New York, 217 pp.

Pemberton, S.G. and Frey, R.W. 1984. Ichnology of storm-influenced shallow marine sequence: Cardium Formation (Upper Cretaceous) at Seebe, Alberta. In: The Mesozoic of middle North America (Eds. D.F. Stott and D.J. Glass). Canadian Society of Petroleum Geologists, Memoir, 9, 281-304.

Pickerill, R.K and Fillion, D. 1983. On the Tremadoc-Arenig and Lower-Upper Tremadoc boundaries in the Bell Island Group, Conception Bay, eastern Newfoundland. Maritime Sediments and Atlantic Geology, 19, 21-30.

Pickerill, R.K. and Narbonne, G.M. 1995. Composite and coumpound ichnotaxa: a case example from the Ordovician of Québec, eastern Canada. Ichnos, 4, 53-69.

Pickerill, R.K., Romano, M. and Meléndez, B. 1984. Arenig trace fossils from the Salamanca area, western Spain. Geological Journal, 19, 249-269.

Raffaelli, D. and Hawkins, S. 1996. Intertidal Ecology. Chapman \& Hall, London, 356 pp.

Ramos, V.A. 1973. Trazas de trilobites de la Formación Campanario (Cámbrico) en la Puna Salteña y otros fósiles problemáticos del Paleozoico inferior. Ameghiniana, 10, 229-234.

Reise, K. 1985. Tidal Flat Ecology. An experimental approach to species interactions. Ecological Studies, 54, Springer-Verlag, Berlin, Heidelberg, 191 pp.

Rindsberg, A. 1999. Workshop on Ichnotaxonomy (WIT), Bornholm, Denmark, August 4-7, 1998. Ichnology Newsletter, 21, 48-50.

Rouault, M. 1850. Note préliminaire sur une nouvelle formation découverte dans le terrain silurien inférieur de la Bretagne. Bulletin de la Société géologique de France, série 2, 7, 724-744.

Sánchez, T.M. 1986. Una fauna de bivalvos en la Formación Santa Gertrudis (Ordovícico) de la Provincia de Salta (Argentina). Ameghiniana, 23, 131-139.

Santisteban, C. and Taberner, C. 1988. Geometry, structure and geodynamics of a sand wave complex in the southeast margin of the Eocene Catalan Basin, Spain. In: Tide-influenced sedimentary environments and facies (Eds. P.L. De Boer, A. van Gelder and S.D. Nio). D. Reidel Publishing Company, Boston, 123-138.
Seilacher, A. 1955. Spuren und Fazies im Unterkambrium. In: Beiträge zur Kenntnis des Kambriums in der Salt Range (Pakistan) (Auts. O.H. Schindewolf and A. Seilacher). Akademie der Wissenschaften und der Literatur in Mainz, mathematisch-naturwissenschaftliche Klasse, Abhandlungen, 10, 117-143.

Seilacher, A. 1962. Form und Funktion des TrilobitenDaktylus. Paläontologische Zeitschrift (H. SchmidtFestband), 218-227.

Seilacher, A. 1970. Cruziana stratigraphy of "nonfossiliferous" Palaeozoic sandstones. In: Trace Fossils (Eds. T.P. Crimes and J.C. Harper). Geological Journal Special Issue, 3, 447-476.

Seilacher, A. 1985. Trilobite palaeobiology and substrate relationship. Transactions of the Royel Society of Edinburgh, 76, 231-237.

Seilacher, A. 1990. Paleozoic trace fossils. In: The Geology of Egypt (Ed. R. Said). A.A. Balkema, Rotterdam, Brookfield, 649-670.

Seilacher, A. 1992. An updated Cruziana stratigraphy of Gondwanan Palaeozoic sandstones. In: The Geology of Libya: volumes IV-VII (Eds. M.J. Salem et al.). Elsevier, Amsterdam, 1565-1580.

Seilacher, A. 1994. How valid is Cruziana stratigraphy? Geologische Rundschau, 83, 752-758.

Seilacher, A. 1996. Evolution of Burrowing Behaviour in Silurian Trilobites: Ichnosubspecies of Cruziana acacensis. In: The Geology of the Sirt Basin: volumes IIII (Ed. M.J. Salem). Elsevier, Amsterdam, 523-530.

Seilacher, A. and Crimes, T.P. 1969. "European" species of trilobite burrows in eastern Newfoundland. In: North Atlantic Geology and Continental Drift (Ed. M. Kay). American Association of Petroleum Geologists Memoir, 12, 145-148.

Simpson, E.L. and Eriksson, K.A. 1991. Depositional facies and controls on parasequence development in siliciclastic tidal deposits from the Lower Proterozoic, upper Mount Guide Quartzite, Mount Isa Inlier, Australia. In: Clastic Tidal Sedimentology (Eds D.G. Smith, G.E. Reinson, B.A. Zaitlin, B.A. and R.A. Rahmani). Canadian Society of Petroleum Geologists, Memoir, 16, 371-387.

Stride, A.H. 1988. Preservation of marine sand wave structures. In: Tide-influenced sedimentary environments and facies (Eds. P.L. De Boer, A. van Gelder and S.D. Nio). D. Reidel Publishing Company, Boston, 13-22.

Swinbanks, D.D. and Murray, J.W. 1981. Biosedimentological zonation of Boundary Bay tidal flats, Fraser River Delta, British Columbia. Sedimentology, 28, 201-237.

Uchman, A. 1998. Taxonomy and ethology of flysch trace fossils: revision of the Marian Ksiąźkiewicz collection and studies of complementary material. Annales Societatis Geologorum Poloniae, 68, 105-218.

Van Wagoner, J.C., Mitchum, R.M., Campion, K.M. and Rahmanian, V.D. 1990. Siliciclastic sequences, stratigraphy in well $\operatorname{logs}$, cores, and outcrops. American Association of Petroleum Geologists, Methods in Exploration Series, 7, 1-55.

Volkheimer, W., Melendi, D.L. y Aceñolaza, F.G. 1980. 
Una microflora ordovícica de la Formación Mojotoro, provincia de Salta. Revista de la Asociación Geológica Argentina, 35, 401-416.

Waisfeld, B.G. 1996. Revisión de la Zona de "Hoekaspis schlagintweiti" Harrington \& Leanza, Ordovícico del noroeste de Argentina. Actas $12^{\circ}$ Congreso Geológico de Bolivia, Tarija, 3, 915-921.

Waisfeld, B.G., Sánchez, T.M. and Carrera, M.G. 1999. Biodiversification Patterns in the Early Ordovician of Argentina. Palaios, 14, 198-214.
Wilson, J.B. 1982. Shelly faunas associated with temperate offshore tidal deposits. In: Offshore tidal sands: processes and deposits (Ed. A.H. Stride). Chapman and Hall, New York, 126-171.

Wilson, J.B. 1986. Faunas of tidal current and wavedominated continental shelves and their use in the recognition of storm deposits. In: Shelf Sands and Sandstone Reservoirs (Eds. R.J. Knight and J.R. McLean). Canadian Society of Petroleum Geologists Memoir, 11, 313-326.

Manuscrito recibido: 15 de febrero, 2000 Manuscrito aceptado: 21 de noviembre, 2000 